\title{
Analysing singularities of a benchmark problem
}

\author{
Teijo Arponen, Institute of Mathematics, \\ Helsinki University of Technology, PL 1100, 02015 TKK, Finland. \\ email: teijo.arponen@hut.fi. \\ Samuli Piipponen, Department of Mathematics, University of Joensuu, \\ PL 111, 80101 Joensuu, Finland. \\ email: samuli.piipponen@joensuu.fi. \\ Jukka Tuomela, Department of Mathematics, University of Joensuu, \\ PL 111, 80101 Joensuu, Finland. \\ email: jukka.tuomela@joensuu.fi.
}

November 20, 2018

\begin{abstract}
The purpose of this paper is to analyze the singularities of a well known benchmark problem "Andrews' squeezing mechanism". We show that for physically relevant parameter values this system admits singularities. The method is based on Gröbner bases computations and ideal decomposition. It is algorithmic and can thus be applied to study constraint singularities which arise in more general situations.
\end{abstract}

Keywords: Multibody systems. Andrews squeezing mechanism. Ideal decomposition. Constraint singularities. Gröbner bases. Descriptor form. Angular coordinates.

Mathematics Subject Classification (AMS) 2000: 70B15, 13P10, 70G25.

\section{Introduction}

The "Andrews' squeezing system" was first described by Giles in Gil78 and further studied in [Man81]. It is a planar multibody system whose topology consists of closed kinematic loops (see Figure 1). The Andrews' system was promoted in [Sch90] as a benchmark problem to compare different multibody solvers. Nowadays it is a well-known benchmark problem [HW91, MI03] for numerical integration of differential-algebraic equations as well. The equations are of the Lagrangian form (or descriptor form, see also Arp01)

$$
\left\{\begin{array}{l}
f\left(t, y, y^{\prime}, y^{\prime \prime}, \lambda\right)=0 \\
g(y)=0
\end{array}\right.
$$

where the function $f$ describes the dynamical equations and $g$ gives the (holonomic) constraints. Here $y \in \mathbb{R}^{n}$ are the (generalized) position coordinates, $y^{\prime}$ and $y^{\prime \prime}$ are the first and second derivatives, respectively, and $\lambda$ is the Lagrange multiplier. 
It is well known that singularities of any kind hinder solving equations numerically [RS88, HW91, BA94, EH95]. Intuitively, a singularity is where the (generic) number of degrees of freedom of the system changes. Mathematically these are the points where the rank of the Jacobian of $g$ drops. Hence in this paper we will not consider the actual dynamical equations and analyse only the constraints given by $g$.

Most differential equation solvers include a possibility to monitor singularities, and usually when proximity of a singularity is detected, the computation is best to be interrupted. But this kind of monitoring is local only, that is, it does not tell us a priori where the singularities lie but only alert us when it is too late to fix things, so to speak. Also, the monitoring is often a non-negligible part of computational cost. Therefore, it would be highly useful to know a priori where the singularities are, or to make sure that there are no singularities, or perhaps even remove them (for the latter approach, see Arp01). Locating singularities has been studied also in [McC00. If we cannot avoid or remove the singularities, at least knowing where they are encountered is helpful (indeed, necessary) when planning the computation without interruptions. One can then tune the chosen integration algorithm such that the disturbing effect of the singularities is diminished, for example by compensating the singularity of the Kepler problem by a local change of variables as in [LR05] within the computation. Further techniques on compensating singularities in multibody systems are gathered and concisely compared in [BA94] and EH95.

The paper is organized as follows: in the next Section we present the situation in detail and formulate the constraint equations in polynomial form. Section 3 gathers the necessary algebraic tools. Section 4 contains the actual analysis where we show that the mechanism indeed has singularities for certain parameter values. In Section 5 there are some numerical examples of singular configurations, and in Section 6 we summarize and discuss the results, and address possible future work.

\section{Andrews' squeezing mechanism}

The squeezing mechanism is given by the following equations.

$$
g(y)=\left\{\begin{array}{l}
a_{1} \cos \left(y_{1}\right)-a_{2} \cos \left(y_{1}+y_{2}\right)-a_{3} \sin \left(y_{3}\right)-b_{1} \\
a_{1} \sin \left(y_{1}\right)-a_{2} \sin \left(y_{1}+y_{2}\right)+a_{3} \cos \left(y_{3}\right)-b_{2} \\
a_{1} \cos \left(y_{1}\right)-a_{2} \cos \left(y_{1}+y_{2}\right)-a_{4} \sin \left(y_{4}+y_{5}\right)-a_{5} \cos \left(y_{5}\right)-w_{1} \\
a_{1} \sin \left(y_{1}\right)-a_{2} \sin \left(y_{1}+y_{2}\right)+a_{4} \cos \left(y_{4}+y_{5}\right)-a_{5} \sin \left(y_{5}\right)-w_{2} \\
a_{1} \cos \left(y_{1}\right)-a_{2} \cos \left(y_{1}+y_{2}\right)-a_{6} \cos \left(y_{6}+y_{7}\right)-a_{7} \sin \left(y_{7}\right)-w_{1} \\
a_{1} \sin \left(y_{1}\right)-a_{2} \sin \left(y_{1}+y_{2}\right)-a_{6} \sin \left(y_{6}+y_{7}\right)+a_{7} \cos \left(y_{7}\right)-w_{2}
\end{array}\right.
$$

Compared to the original articles mentioned above, we have chosen the following notation for the parameters and angles:

$$
\begin{array}{lllllll}
a_{1}=r r & a_{2}=d & a_{3}=s s & a_{4}=e & a_{5}=z t & a_{6}=z f & a_{7}=u \\
b_{1}=x b & b_{2}=y b & w_{1}=x a & w_{2}=y a \\
y_{1}=\beta & y_{2}=\Theta & y_{3}=\gamma & y_{4}=\Phi & y_{5}=\delta & y_{6}=\Omega \quad y_{7}=\epsilon
\end{array}
$$

so the positions in Cartesian coordinates of the fixed nodes $A$ and $B$ are given by $b=$ $\left(b_{1}, b_{2}\right)$ and $w=\left(w_{1}, w_{2}\right)$, and the lengths of the rods by $a=\left(a_{1}, \ldots, a_{7}\right)$, see Figures 1 and 2 . 
Fixing the parameters $a, b$, and $w$, we have a map $g: \mathbb{R}^{7} \rightarrow \mathbb{R}^{6}$. Hence the set of possible configurations, which is the zeroset $M_{g}=g^{-1}(0)$, is in general a curve (or possibly empty). Our task is to analyse the singularities of $M_{g}$, so let us state more precisely what is meant by a singularity. As mentioned before, in a singularity the number of degrees of freedom changes. It is well known [RS88, BA94, McC00] that this corresponds to the situation where the rank of Jacobian drops.

Definition 2.1. Let $f: \mathbb{R}^{n} \rightarrow \mathbb{R}^{k}$ be any smooth map where $k<n$ and let $d f$ be its Jacobian matrix. Let $M=f^{-1}(0) \subset \mathbb{R}^{n}$ be the zeroset of $f$. A point $q \in M$ is a singular point of $M$, if $d f$ does not have maximal rank at $q$.

What in fact geometrically "happens" at a singular point may be quite complicated to determine. Typically the tangent space to $M$ does not change continuously in the neighbourhood of a singular point, or possibly $M$ intersects itself there. However, in all cases numerical problems occur, so it is important to try to find all singular points.

Note that the constraint equations (2) (and hence the elements of its Jacobian matrix) are not polynomials, yet our algebraic approach works only in a polynomial setting. However, this problem is circumvented by reformulating $g(y)$ as polynomials in the sines and cosines of $y_{i}$ by using the trigonometric identities

$$
\begin{aligned}
& \cos (x)^{2}+\sin (x)^{2}=1 \\
& \sin (x \pm y)=\sin (x) \cos (y) \pm \cos (x) \sin (y) \\
& \cos (x \pm y)=\cos (x) \cos (y) \mp \sin (x) \sin (y)
\end{aligned}
$$

Setting $c_{i}=\cos \left(y_{i}\right), \quad s_{i}=\sin \left(y_{i}\right)$ we get the equations

$$
p(c, s)=\left\{\begin{array}{l}
a_{1} c_{1}-a_{2}\left(c_{1} c_{2}-s_{1} s_{2}\right)-a_{3} s_{3}-b_{1}=0 \\
a_{1} s_{1}-a_{2}\left(s_{1} c_{2}+c_{1} s_{2}\right)+a_{3} c_{3}-b_{2}=0 \\
a_{1} c_{1}-a_{2}\left(c_{1} c_{2}-s_{1} s_{2}\right)-a_{4}\left(s_{4} c_{5}+c_{4} s_{5}\right)-a_{5} c_{5}-w_{1}=0 \\
a_{1} s_{1}-a_{2}\left(s_{1} c_{2}+c_{1} s_{2}\right)+a_{4}\left(c_{4} c_{5}-s_{4} s_{5}\right)-a_{5} s_{5}-w_{2}=0 \\
a_{1} c_{1}-a_{2}\left(c_{1} c_{2}-s_{1} s_{2}\right)-a_{6}\left(c_{6} c_{7}-s_{6} s_{7}\right)-a_{7} s_{7}-w_{1}=0 \\
a_{1} s_{1}-a_{2}\left(s_{1} c_{2}+c_{1} s_{2}\right)-a_{6}\left(s_{6} c_{7}+c_{6} s_{7}\right)+a_{7} c_{7}-w_{2}=0 \\
c_{i}^{2}+s_{i}^{2}-1=0, \quad i=1, \ldots, 7 .
\end{array}\right.
$$

We have 13 polynomial equations $\left(p_{i}=0\right), 11$ parameters $\left(a_{1}, \ldots, a_{7}, b_{1}, b_{2}, w_{1}, w_{2}\right)$ and 14 variables $\left(c_{1}, s_{1}, \ldots, c_{7}, s_{7}\right)$. Note that each $p_{i}$ is of degree two in $c_{i}, s_{i}$. The equations $p_{1}=0, \ldots, p_{6}=0$ correspond directly to the 6 original equations $g(y)=0$ with the simple substitutions above (for example $\cos \left(y_{1}+y_{2}\right)=c_{1} c_{2}-s_{1} s_{2}$ ) and the equations $p_{7}=0, \ldots, p_{13}=0$ are the extra identities due to "forgetting" the angle variables $y_{i}$.

Note that this reformulation of the constraints as algebraic equations is not just a trick which happens to work in this special case; indeed most constraints appearing in the simulation of multibody systems are of this type.

Now the above equations define $p$ as a map $p: \mathbb{R}^{14} \rightarrow \mathbb{R}^{13}$. Hence we expect that the zeroset $V=p^{-1}(0) \subset \mathbb{R}^{14}$ is a curve (or possibly empty). Singularities are then the points of this curve where the rank of $d p$ is not maximal. To find these points we need now to introduce some tools from commutative algebra. 


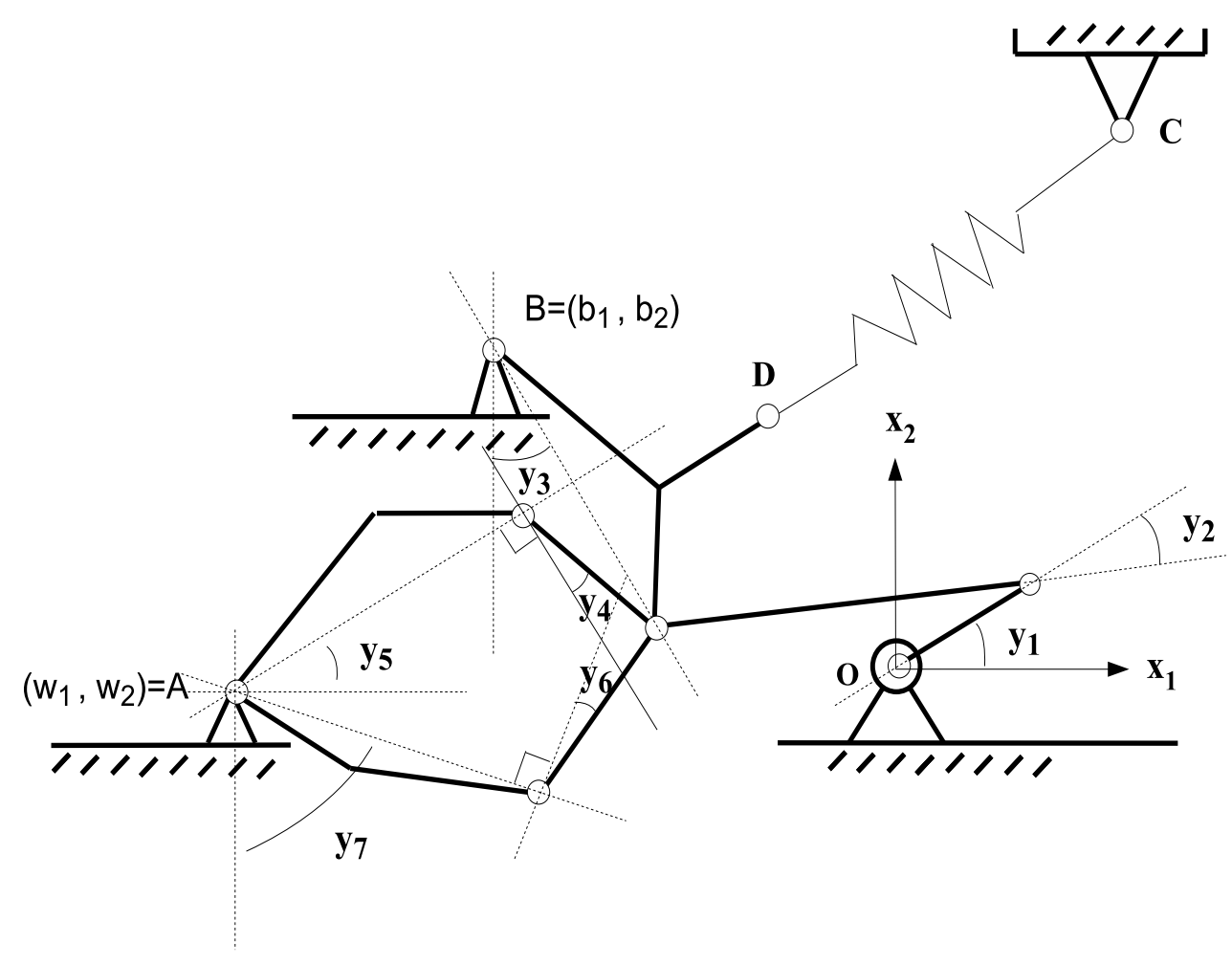

Figure 1: The angles $y_{i}$ of the Andrews' system.

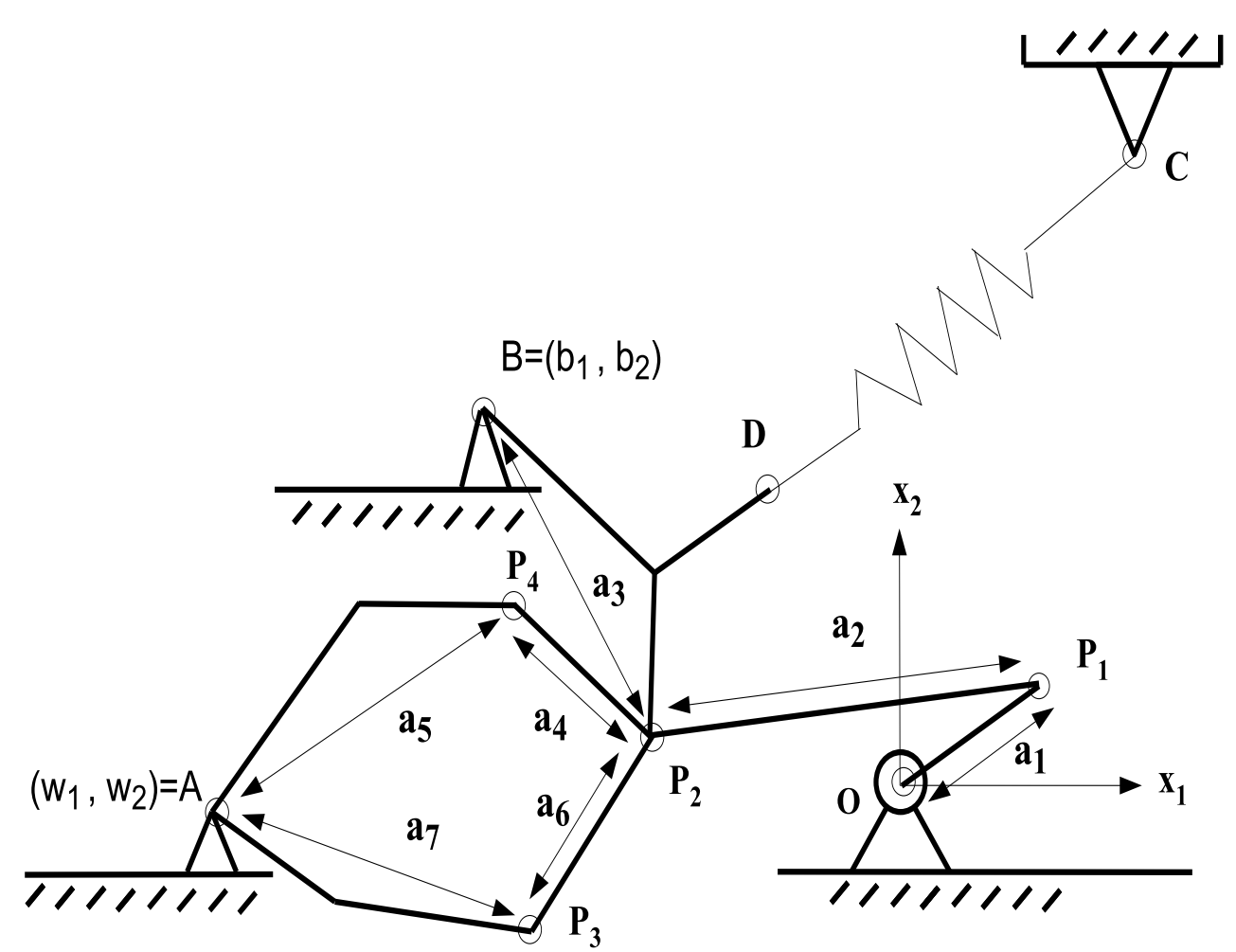

Figure 2: The lengths $a_{i}$ and nodes of the Andrews' system. 


\section{Background}

In this section we present briefly the necessary definitions from commutative algebra and algebraic geometry. More details can be found in [CLO92], GP02], [Nor76], and [Eis96]. These are roughly in the order of increasing difficulty, [CLO92] being the most accessible, but unfortunately not containing the necessary material on the Fitting ideals.

\subsection{Ideals and varieties}

Let $\mathbb{K}$ be an algebraic field and let $\mathbb{K}\left[x_{1}, \ldots, x_{n}\right]$ be the ring of polynomials in $x_{1}, \ldots, x_{n}$, with coefficients in $\mathbb{K}$. A subset $I \subset \mathbb{K}\left[x_{1}, \ldots, x_{n}\right]$ is an ideal if it satisfies

(i) $0 \in I$.

(ii) If $f, g \in I$, then $f+g \in I$.

(iii) If $f \in I$ and $h \in \mathbb{K}\left[x_{1}, \ldots, x_{n}\right]$, then $h f \in I$.

Ideals are often given by generators. Let $f_{1}, \ldots, f_{s} \in \mathbb{K}\left[x_{1}, \ldots, x_{n}\right]$. Then the set

$$
\left\langle f_{1}, \ldots, f_{s}\right\rangle:=\left\{\sum_{i=1}^{s} h_{i} f_{i} \mid h_{1}, \ldots, h_{s} \in \mathbb{K}\left[x_{1}, \ldots, x_{n}\right]\right\}
$$

is an ideal generated by $f_{1}, \ldots, f_{s}$. Any set of generators is called a basis.

Ideals are purely algebraic objects. The geometrical counterpart of an ideal is its locus, or variety. Let $I$ be an ideal in $\mathbb{K}\left[x_{1}, \ldots, x_{n}\right]$. Its corresponding variety is

$$
\bigvee_{\mathbb{F}}(I)=\left\{\left(a_{1}, \ldots, a_{n}\right) \in \mathbb{F}^{n} \mid f\left(a_{1}, \ldots, a_{n}\right)=0 \quad \forall f \in I\right\}
$$

where $\mathbb{F}$ is some field extension of $\mathbb{K}$. Note that it is often natural to choose $\mathbb{F}$ different from $\mathbb{K}$. If the field is clear from context we will sometimes write simply $\vee(I)$.

Now different ideals may have the same variety. However, if one is interested mainly in the variety then it is useful to define

$$
\sqrt{I}=\left\{f \in \mathbb{K}\left[x_{1}, \ldots, x_{n}\right] \mid f^{n} \in I \text { for some } n \geq 1\right\} .
$$

If $I$ is an ideal, then $\sqrt{I}$ is the radical of $I$; it is the biggest ideal that has the same variety as $I$ and all ideals having the same variety have the same radical. Also, always $I \subset \sqrt{I}$ and if $I=\sqrt{I}$ we say that $I$ is a radical ideal. Some rudimentary properties among ideals and their varieties are in the following

Lemma 3.1. Let $I$ and $J$ be ideals. Then

1. $\mathrm{V}(I \cup J)=\mathrm{V}(I) \cap \mathrm{V}(J)$.

2. $\mathrm{V}(I \cap J)=\mathrm{V}(I) \cup \mathrm{V}(J)$.

3. $I \subset J$ if and only if $\mathrm{\vee}(I) \supset \mathrm{V}(J)$.

Next we have to express the rank condition algebraically. To this end we need

Definition 3.1. If $I=\left\langle f_{1}, \ldots, f_{s}\right\rangle$, its Fitting ideal $F_{I}$ is the ideal generated by all maximal minors of the Jacobian matrix of $\left(f_{1}, \ldots, f_{s}\right)$ 1]

\footnotetext{
${ }^{1}$ In general one can define Fitting ideals of minors of any given size. However, the above definition is sufficient for purposes of the present paper.
} 
Now $\mathrm{V}\left(F_{I}\right)$ corresponds to the points where the rank is not maximal. However, the points are required also to be on $\mathrm{V}(I)$. Hence we conclude that the set of singular points, $S$, is given by

$$
S=\mathrm{V}\left(I \cup F_{I}\right)
$$

In analysing varieties it is often helpful to decompose them to simpler parts. Similarly one may try to decompose a given ideal to simpler parts. This leads to following notions.

Definition 3.2. A variety $V$ is irreducible if $V=V_{1} \cup V_{2}$ implies $V=V_{1}$ or $V=V_{2}$. An ideal $I$ is prime if $f, g \in \mathbb{K}\left[x_{1}, \ldots, x_{n}\right]$ and $f g \in I$ imply that either $f \in I$ or $g \in I$.

There is a very close connection between prime ideals and irreducible varieties. The precise nature of this depends on the chosen field. However, for our purposes the following is sufficient.

Lemma 3.2. If $I$ is prime, then $\mathrm{V}(I)$ is irreducible.

Any radical ideal can be written uniquely as a finite intersection of prime ideals,

$$
\sqrt{I}=I_{1} \cap \cdots \cap I_{r}
$$

where $I_{i} \not \subset I_{j}$ for $i \neq j$.

This is known as the prime decomposition of $\sqrt{I}$ and the $I_{i}$ 's are called the minimal associated primes of $I$. The above Lemma then immediately gives:

\section{Corollary 3.1.}

$$
\mathrm{V}(I)=\mathrm{V}(\sqrt{I})=\mathrm{V}\left(I_{1}\right) \cup \cdots \cup \mathrm{V}\left(I_{r}\right)
$$

where all $\mathrm{V}\left(I_{i}\right)$ are irreducible.

Hence our strategy in analysing varieties is to compute the minimal associated primes of the relevant ideal, and then examine each irreducible component separately.

\subsection{Gröbner bases}

An essential thing is that all the operations above, especially finding the radical and the prime decomposition can be computed algorithmically using the given generators of $I$. To do this we need to compute special bases for ideals, called Gröbner bases. We will only briefly indicate the relevant ideas and refer to [CLO92 and GP02 for more details.

First we need to introduce monomial orderings. All the algorithms handling the ideals are based on some orderings among the terms of the generators of the ideal.

Intuitively, an ordering $\succ$ is such that given a set of monomials (e.g. terms of a given polynomial), $\succ$ puts them in order of importance: given any two monomials $x^{\alpha}:=$ $x_{1}^{\alpha_{1}} \ldots x_{n}^{\alpha_{n}}$ and $x^{\beta}$, where $\alpha \neq \beta$ are different multi-indices, then either $x^{\alpha} \succ x^{\beta}$ or $x^{\beta} \succ x^{\alpha}$. A common choice is to use degree reversed lexicographic ordering [CLO92]. In our analysis we shall frequently need product orders, which are formed as follows: if $\succ_{A}$ and $\succ_{B}$ are two orderings, we shall divide the variables $x_{i}$ into two subsets, and use $\succ_{A}$ on the first subset and $\succ_{B}$ on the second. This is indicated with the following notation:

$$
\mathbb{K}\left[\left(x_{4}, x_{5}, x_{7}\right),\left(x_{1}, x_{2}, x_{3}, x_{6}\right)\right] .
$$

This is the same set as $\mathbb{K}\left[x_{1}, \ldots, x_{7}\right]$ but now the parenthesis indicate that we will use $\succ_{A}$ among the variables $\left(x_{4}, x_{5}, x_{7}\right)$, and $\succ_{B}$ among the variables $\left(x_{1}, x_{2}, x_{3}, x_{6}\right)$, and moreover 
all monomials where variables of the first group appear are always bigger than monomials where there are only variables of the second group. We will see later why this is useful.

Finally, the aforementioned Gröbner basis is a special kind of generating set, with respect to some ordering. Given any set of generators and an ordering, the corresponding Gröbner basis exists and can be computed. The relevant algorithm is usually called the Buchberger algorithm. The drawback of this algorithm is that it has a very high complexity in the worst case, and in practice the complexity depends quite much on the chosen ordering 2

Anyway Gröbner bases have proved to be very useful in many different applications. Nowadays there exist many different implementations and improvements of the Buchberger algorithm. We chose to use the well-known program Singular [GPS05], GP02] in all the computations in this paper.

\section{Analysing singularities}

\subsection{Geometric description of the singularities}

Now getting back to our system (3) we see that we can take the components of $p$ to be elements of $\mathbb{Q}(a, b, w)[c, s]$ where $\mathbb{Q}(a, b, w)$ is the field of rational functions of $a, b$, and $w$. Hence we have an ideal $J=\left\langle p_{1}, \ldots, p_{13}\right\rangle \subset \mathbb{Q}(a, b, w)[c, s]$ and the corresponding Fitting ideal $F_{J}$. On the other hand we may view the "parameters" $a, b$, and $w$ also as variables since they appear polynomially in the equations; hence we could also consider $J \subset \mathbb{Q}[a, b, w, c, s]$. Taking this point of view we can give an intuitive description of what kind of situations we can expect.

$$
\left\{\begin{array}{l}
J \subset \mathbb{Q}[a, b, w, c, s] \\
V_{\mathbb{R}}(J) \subset \mathbb{R}^{25} .
\end{array}\right.
$$

In this way $V_{\mathbb{R}}(J)$ should be 12 dimensional (recall $J$ is generated by 13 equations), i.e. a curve depending on 11 parameters. On the other hand if we fix parameters $a, b$, and $w$ we get a curve in $\mathbb{R}^{14}$ which will be denoted by $V_{a, b, w}$. In the same way we can view $V_{\mathbb{R}}\left(J \cup F_{J}\right)$ as a variety in $\mathbb{R}^{25}$, and fixing the parameters we get the singular points $V_{a, b, w}^{S}$. Obviously $V_{a, b, w}^{S} \subset V_{a, b, w} \subset \mathbb{R}^{14}$.

Then what kind of variety should $V_{\mathbb{R}}\left(J \cup F_{J}\right)$ be? Since the Jacobian of $p$ is of size $13 \times 14$, generically we expect to get 2 independent conditions in order the rank to drop. That is, augmenting $J$ with $F_{J}$ should bring in 2 more equations. Hence we expect that $V_{\mathbb{R}}\left(J \cup F_{J}\right)$ is 10 dimensional; in other words we expect that if 11 parameters are chosen independently then $V_{a, b, w}^{S}$ should be empty. On the other hand if a single condition among parameters is satisfied, then $V_{a, b, w}^{S}$ should consist of isolated points.

Further, if there are 2 conditions among parameters (i.e. 9 parameters freely chosen), then it would be possible that $V_{a, b, w}^{S}$ were one dimensional. But then our original constraint equations would be redundant, i.e. there would be more than one degree of freedom.

Below we will in fact observe that if a certain condition on parameters is satisfied, $V_{a, b, w}^{S}$ is indeed a finite set of points.

\footnotetext{
${ }^{2}$ So far, no satisfactory theory of Gröbner basis complexity has been done.
} 


\subsection{Singular variety}

To study $V_{\mathbb{R}}\left(J \cup F_{J}\right)$ we could in principle use Gröbner basis theory in a straightforward manner. Let $G$ be the Gröbner basis of $J \cup F_{J}$ using the product order $\mathbb{Q}[(c, s),(a, b, w)]$. Let us denote by $g_{1}, \ldots, g_{r}$ the elements of $G$ which do not depend on $c$ and $s$.

Definition 4.1. Let $S_{J}=\left\langle g_{1}, \ldots, g_{r}\right\rangle$; then we say that $\bigvee_{\mathbb{R}}\left(S_{J}\right) \subset \mathbb{R}^{11}$ is the singular variety associated to $J$.

It follows from the Gröbner basis theory that $V_{a, b, w}$ can have singularities only if $(a, b, w) \in \mathrm{V}_{\mathbb{R}}\left(S_{J}\right)$. Hence theoretically, we could now find the singularities of the Andrews' system in a straightforward manner by calculating the Gröbner basis of $J \cup F_{J}$. But this is an enormous task, due to $F_{J}$ being generated by high degree polynomials, not to mention including the 11 parameters $a, b, w$. We could not get the solution in a finite time using our work station with $64 \mathrm{~GB}$ memory.

Instead, something else needs to be done. Luckily there is another approach: noting that $p_{1}, p_{3}, p_{5}$ have common terms, as well as $p_{2}, p_{4}, p_{6}$, gives us motivation to study two subsystems. One spanned by $p_{5}-p_{3}$ and $p_{6}-p_{4}$, the other one spanned by $p_{5}-p_{1}$ and $p_{6}-p_{2}$ (along with the relevant trigonometric identities from $p_{7}, \ldots, p_{13}$ ). These subsystems are handleable and give useful information for the whole system as well. Proceeding in this way we could at least determine that the singular variety is not empty and we could compute some subvarieties of it.

\subsection{Subsystem 4567}

Intuitively, the nodes and bars 4, 5, 6, 7 formulate a subsystem, see Figures 1 and 2, We suspect that when the lengths $a_{4}, \ldots, a_{7}$ are such that the " 4567 " system is able to become one-dimensional, hence in some sense degenerated, there should be a singularity in the whole system (see also the net example in Arp01]). We will shortly see that this is indeed the case.

Define

$$
\begin{aligned}
q_{1} & :=p_{5}-p_{3}=a_{4}\left(s_{4} c_{5}+c_{4} s_{5}\right)+a_{5} c_{5}-a_{6}\left(c_{6} c_{7}-s_{6} s_{7}\right)-a_{7} s_{7} \\
q_{2} & :=p_{4}-p_{6}=a_{4}\left(c_{4} c_{5}-s_{4} s_{5}\right)-a_{5} s_{5}+a_{6}\left(s_{6} c_{7}+c_{6} s_{7}\right)-a_{7} c_{7} \\
q_{i} & :=p_{i+7}=c_{i+1}^{2}+s_{i+1}^{2}-1, \quad i=3, \ldots, 6 .
\end{aligned}
$$

Note that $q_{1}, q_{2}$ contain only angles $c_{i}, s_{i}$ and parameters $a_{i}$ for $i=4, \ldots, 7$. That is why we do not need the other $p_{i}$ 's. Let $J_{4567}$ be the ideal spanned by $q_{1}, \ldots, q_{6}$. Hence we have

$$
J_{4567} \subset \mathbb{Q}\left[\left(c_{4}, s_{4}, c_{5}, s_{5}, c_{6}, s_{6}, c_{7}, s_{7}\right),\left(a_{4}, a_{5}, a_{6}, a_{7}\right)\right]
$$

where we have indicated the relevant product order. The Gröbner basis $G$ for $J_{4567} \cup F_{J_{4567}}$ with respect to this ordering contains 191 elements (denoted by $g_{1}, \ldots, g_{191}$ ), out of which 
3 are especially enlightening:

$$
\begin{aligned}
g_{5} & =c_{6} a_{6} a_{7}, \\
g_{16} & =c_{4} a_{4} a_{5}, \quad \text { and } \\
g_{1} & =\prod_{i=1}^{8} t_{i}, \quad \text { where } \\
t_{1} & =a_{4}-a_{5}-a_{6}-a_{7} \\
t_{2} & =a_{4}-a_{5}+a_{6}+a_{7} \\
t_{3} & =a_{4}+a_{5}+a_{6}+a_{7} \\
t_{4} & =a_{4}+a_{5}-a_{6}-a_{7} \\
t_{5} & =a_{4}-a_{5}+a_{6}-a_{7} \\
t_{6} & =a_{4}-a_{5}-a_{6}+a_{7} \\
t_{7} & =a_{4}+a_{5}-a_{6}+a_{7} \\
t_{8} & =a_{4}+a_{5}+a_{6}-a_{7} .
\end{aligned}
$$

Since $g_{1}$ is the only generator which does not contain any variables $c_{i}$ and $s_{i}$ we conclude that

Theorem 1. The singular variety of $J_{4567}$ is

$$
S_{J_{4567}}=\mathrm{V}\left(\left\langle g_{1}\right\rangle\right) .
$$

Note that the factorization of $g_{1}$ gives us the prime decomposition of $\left\langle g_{1}\right\rangle$ and hence decomposition of $\mathrm{V}\left(\left\langle g_{1}\right\rangle\right)$ into 8 linear irreducible varieties.

Our next task is to show that at least some points of the singular variety extend to actual (physically relevant) singularities of the whole system. Recall that each generator $g_{i}$ corresponds to an equation $g_{i}=0$. Since $a_{i}>0$ in physically relevant cases, generators $g_{5}$ and $g_{16}$ imply that all the singularities of $J_{4567}$ have necessarily $c_{6}=c_{4}=0$ (conditions for the angles 4 and 6 ). In other words, in ideal-theoretic language, we can as well study the ideal

$$
T:=\left\langle J_{4567}, F_{J_{4567}}, c_{4}, c_{6}\right\rangle .
$$

Now the prime decomposition of $\sqrt{T}$ has 16 components:

$$
\sqrt{T}=T_{1} \cap \ldots \cap T_{16} .
$$

Inspecting the generators of each of $T_{j}$, it is noticed that every $T_{j}$ contains the $t_{i}$ 's or $a_{i}$ 's. Recall that a generator $a_{i}$ in an ideal corresponds in the variety to a condition $a_{i}=0$ which is non-physical. Moreover, $t_{3}$ is now a non-physical condition contradicting $a_{i}>0 \forall i$. Hence we discard (as in Arp01]) those ideals which have a non-physical generator that would imply $a_{i} \leq 0$ for some $i$, and we are left with 7 ideals, whose generators are:

$$
\begin{aligned}
& T_{1}=\left\langle c_{7}^{2}+s_{7}^{2}-1, t_{1}, s_{6}+1, s_{5}-c_{7}, c_{5}+s_{7}, s_{4}+1, c_{4}, c_{6}\right\rangle \\
& T_{2}=\left\langle c_{7}^{2}+s_{7}^{2}-1, t_{2}, s_{6}+1, s_{5}+c_{7}, c_{5}-s_{7}, s_{4}+1, c_{4}, c_{6}\right\rangle \\
& T_{3}=\left\langle c_{7}^{2}+s_{7}^{2}-1, t_{4}, s_{6}+1, s_{5}+c_{7}, c_{5}-s_{7}, s_{4}-1, c_{4}, c_{6}\right\rangle \\
& T_{4}=\left\langle c_{7}^{2}+s_{7}^{2}-1, t_{5}, s_{6}-1, s_{5}-c_{7}, c_{5}+s_{7}, s_{4}+1, c_{4}, c_{6}\right\rangle \\
& T_{5}=\left\langle c_{7}^{2}+s_{7}^{2}-1, t_{6}, s_{6}-1, s_{5}+c_{7}, c_{5}-s_{7}, s_{4}+1, c_{4}, c_{6}\right\rangle \\
& T_{6}=\left\langle c_{7}^{2}+s_{7}^{2}-1, t_{7}, s_{6}-1, s_{5}-c_{7}, c_{5}+s_{7}, s_{4}-1, c_{4}, c_{6}\right\rangle \\
& T_{7}=\left\langle c_{7}^{2}+s_{7}^{2}-1, t_{8}, s_{6}-1, s_{5}+c_{7}, c_{5}-s_{7}, s_{4}-1, c_{4}, c_{6}\right\rangle .
\end{aligned}
$$


Especially, we see that $s_{6}= \pm 1, s_{5}= \pm c_{7}, c_{5}= \pm s_{7}$, and $s_{4}= \pm 1$. Now we are ready to continue with the original system $J \cup F_{J}$.

Remark 4.1. Mathematically speaking the analyses of all cases $T_{i}$ are completely similar. However, on physical grounds the cases $T_{1}, T_{2}, T_{6}$ and $T_{7}$ are not so interesting. Indeed, in these cases the length of one of the rods corresponding to $a_{4}, a_{5}, a_{6}$ and $a_{7}$ is equal to the sum of the lengths of three others. Hence all four rods could be modelled as a single rod which would make the whole model significantly simpler. In the remaining cases no such reduction can be done, and we chose to examine the ideal $T_{5}$ in detail. See also remark 4.3 .

The case $T_{5}$ gives us conditions $s_{4}=-1, s_{6}=1, s_{5}=-c_{7}, c_{5}=s_{7}$, and $a_{7}=a_{5}+a_{6}-a_{4}$ which we substitute into the original system. Next we will show that the resulting system has real solutions. These will be the required singular points.

The above substitutions simplify the generators of $J \cup F_{J}$ so that we get the following ideal:

$$
\begin{aligned}
& K=\left\langle K_{1} \cup K_{2}\right\rangle, \\
& K_{1}:\left\{\begin{array}{l}
k_{1}=a_{2}\left(-c_{1} c_{2}+s_{1} s_{2}\right)+c_{1} a_{1}-s_{3} a_{3}-b_{1} \\
k_{2}=a_{2}\left(-s_{1} c_{2}-c_{1} s_{2}\right)+s_{1} a_{1}+c_{3} a_{3}-b_{2} \\
k_{3}=c_{1}^{2}+s_{1}^{2}-1 \\
k_{4}=c_{2}^{2}+s_{2}^{2}-1,
\end{array}\right. \\
& K_{2}:\left\{\begin{array}{l}
k_{5}=s_{7}\left(a_{4}-a_{5}\right)+s_{3} a_{3}+b_{1}-w_{1} \\
k_{6}=c_{7}\left(a_{5}-a_{4}\right)-c_{3} a_{3}+b_{2}-w_{2} \\
k_{7}=c_{3}^{2}+s_{3}^{2}-1 \\
k_{8}=c_{7}^{2}+s_{7}^{2}-1 .
\end{array}\right.
\end{aligned}
$$

In $K_{2}$ we have 4 equations for 4 unknowns $c_{3}, s_{3}, c_{7}$, and $s_{7}$; hence it appears reasonable that we can get a finite number of solutions. Then we can substitute the computed values to $K_{1}$ which then becomes also a system of 4 equations for 4 unknowns $c_{1}, s_{1}, c_{2}$, and $s_{2}$. By the same reasoning we again expect that it is possible to get some solutions for appropriate parameter values.

We could numerically solve the variables from these equations (and, indeed, we will, in the numerical examples), but to analyze the situation in more detail we need to study these further.

Then starting with the system $K_{2}$ we solve the angles 3 and 7 by the following trick. First we inspect the ideal generated by $K_{2}$ in the ring

$$
\mathbb{Q}\left(b_{1}, b_{2}, w_{1}, w_{2}, a_{3}, a_{4}, a_{5}\right)\left[c_{3}, s_{3}, c_{7}, s_{7}\right] .
$$

Calculating the Gröbner basis $\tilde{G}$ of $\left\langle K_{2}\right\rangle$ with respect to the lexicographic ordering we get 4 generators:

$$
\begin{aligned}
& \tilde{g}_{1}=f_{1} s_{7}^{2}+f_{2} s_{7}-f_{3} f_{4} \\
& \tilde{g}_{2}=2\left(b_{2}-w_{2}\right)\left(a_{4}-a_{5}\right) c_{7}-2\left(b_{1}-w_{1}\right)\left(a_{4}-a_{5}\right) s_{7}+f_{5}=0 \\
& \tilde{g}_{3}=a_{3} s_{3}+\left(a_{4}-a_{5}\right) s_{7}+b_{1}-w_{1}=0 \\
& \tilde{g}_{4}=a_{3} c_{3}+\left(a_{4}-a_{5}\right) c_{7}+w_{2}-b_{2}=0 .
\end{aligned}
$$

where the auxiliary expressions $f_{i}$ are lengthy combinations of the parameters $a_{i}, b_{i}$ (see the appendix) 3

\footnotetext{
${ }^{3}$ The algorithms actually give by default only sums of monomials instead of products like $2\left(b_{2}-\right.$
} 
Now $\tilde{g}_{1}$ contains only $s_{7}$ and parameters. Note that $f_{1}=0$ if and only if $a_{4}=a_{5}$. Assuming $a_{4} \neq a_{5}$ the equation $\tilde{g}_{1}=0$ is a polynomial in $s_{7}$ of degree 2 , hence in order to have real solutions we need to impose the condition

$$
f_{2}^{2}+4 f_{1} f_{3} f_{4} \geq 0
$$

This condition can easily be checked when the parameters $a, b, w$ have been given numerical values. Once $s_{7}$ is known, $c_{7}, s_{3}, c_{3}$ can be solved from the linear equations of $\tilde{G}$, provided $a_{4} \neq a_{5}$ and $w_{2} \neq b_{2}$.

The cases $w_{2}=b_{2}$ and/or $a_{4}=a_{5}$ can be summarized as follows:

(i) If $w_{2}=b_{2}$ but $a_{4} \neq a_{5}$, we still get equations similar to $\tilde{G}$, but now $s_{3}$ has a quadratic equation instead of $s_{7}$.

(ii) If $a_{4}=a_{5}$, the system typically does not have solutions. At least, a further condition among parameters, namely $|b-w|=a_{3}$, arises. We shall not elaborate this nongeneric behaviour further. In Section 4.5 .2 we consider an example of this situation.

Remark 4.2. In general, when the inequality in (8) is strict, $s_{7}$ has 2 possible values. Therefore, the tuples $\left(s_{3}, c_{3}, s_{7}, c_{7}\right)$ have in general 2 possible values because the other ones in the tuple are determined uniquely from $s_{7}$.

The only thing left to be done, in this $J_{4567}$ subsystem case, is to solve $c_{1}, s_{1}, c_{2}, s_{2}$. This is done with the ideal $\left\langle K_{1}\right\rangle$ given in (6).

Remark 4.3. Had we used any other $T_{i}$ instead of $T_{5}$ above, we would have ended up with this same ideal $\left\langle K_{1}\right\rangle$.

We calculate the Gröbner basis $\hat{G}$ of $\left\langle K_{1}\right\rangle$, this time in the ring

$$
\mathbb{Q}\left(a_{1}, a_{2}, a_{3}, b_{1}, b_{2}, c_{3}, s_{3}\right)\left[c_{1}, s_{1}, c_{2}, s_{2}\right] .
$$

Note especially that $s_{3}, c_{3}$ are here treated as parameters, due to being now known expressions in the parameters $a, b, w$. We again use lexicographic ordering and get 4 generators $\hat{g}_{1}, \ldots, \hat{g}_{4}$. Analogously to $s_{7}$ above, now for $s_{2}$ we get the second degree polynomial equation

$$
\hat{g}_{1}=\left(-4 a_{1}^{2} a_{2}^{2}\right) s_{2}^{2}-n_{1} n_{2}=0
$$

where

$$
\begin{aligned}
& n_{1}=a_{1}^{2}+2 a_{1} a_{2}+a_{2}^{2}-a_{3}^{2}-2 a_{3} b_{1} s_{3}+2 a_{3} b_{2} c_{3}-b_{1}^{2}-b_{2}^{2} \\
& n_{2}=a_{1}^{2}-2 a_{1} a_{2}+a_{2}^{2}-a_{3}^{2}-2 a_{3} b_{1} s_{3}+2 a_{3} b_{2} c_{3}-b_{1}^{2}-b_{2}^{2}
\end{aligned}
$$

and linear equations for $c_{2}, s_{1}, c_{1}$ :

$$
\begin{aligned}
& \hat{g}_{2}=d_{1} c_{2}+d_{2}+d_{3} \\
& \hat{g}_{3}=l_{1} s_{1}+l_{2}+l_{3} \\
& \hat{g}_{4}=\left(a_{1}^{2}-a_{2}^{2}\right) c_{1}+l_{4}
\end{aligned}
$$

where the auxiliary expressions $d_{i}, l_{i}$ are certain known (but lengthy) functions of $a, b$, apart from $l_{4}$ which depends on $s_{1}, s_{2}, c_{2}$ as well. (See the appendix.) In order to have real solutions for $s_{2}$, (9) implies the condition

$$
E:=n_{1} n_{2} \leq 0
$$

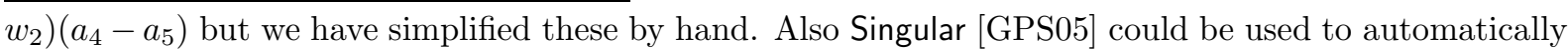
factorize into products but would involve some more elaborate programming. 
These $\hat{g}_{i}$ determine $s_{2}, c_{2}, s_{1}, c_{1}$ provided $d_{1} \neq 0, l_{1} \neq 0, a_{1} \neq a_{2}$. To analyse the cases $d_{1}=0, a_{1}=a_{2}$, and/or $l_{1}=0$, it is helpful to define

$$
d_{0}:=a_{3}^{2}+2 a_{3} b_{1} s_{3}-2 a_{3} b_{2} c_{3}+b_{1}^{2}+b_{2}^{2} .
$$

It turns out that $l_{1}=0 \Leftrightarrow d_{1}=0 \Leftrightarrow d_{0}=0$. After rearranging the terms (see the appendix) it can be seen that the condition (10) is equivalent to

$$
\left(a_{1}-a_{2}\right)^{2} \leq d_{0} \leq\left(a_{1}+a_{2}\right)^{2} .
$$

Therefore, if $a_{1} \neq a_{2}$ then $d_{0} \neq 0$ and the equations above can be solved. The case $a_{1}=a_{2}$, $d_{0} \neq 0$ does not essentially change the situation: we still have a quadratic equation for $s_{2}$, and linear ones for the others, with a different coefficient for $c_{1}$.

The remaining case $a_{1}=a_{2}, d_{0}=0$ corresponds to the situation where the centre node coincides with the origin. This gives another singularity (the angle $y_{1}$ remains arbitrary) but is a rather special case and will not be pursued further here.

Theorem 2. Let us suppose that the parameters $a, b, w$ satisfy the following conditions: $a_{4} \neq a_{5}$ and

$$
\begin{aligned}
& n_{1}\left(4 a_{1} a_{2}-n_{1}\right) \geq 0 \\
& f_{2}^{2}+16\left(a_{4}-a_{5}\right)^{2}|b-w|^{2} f_{3} f_{4} \geq 0
\end{aligned}
$$

Then $V_{a, b, w}$ contains at least 2 singular points. If the inequalities are strict we get in general at least 4 singular points.

It may appear that we also have at most 4 singular points. However, it is a priori possible that the other systems $T_{i}$ yield more singular points with the same parameter values.

Proof. The first part of the theorem merely collects what we have shown above, with the simplifications $n_{2}=n_{1}-4 a_{1} a_{2}$ and $f_{1}=4\left(a_{4}-a_{5}\right)^{2}|b-w|^{2}$. The conditions are due to univariate second degree polynomial equations, which have real solutions if and only if (8) and (10) (for $s_{7}$ and $s_{2}$, respectively) are fulfilled. The other variables are determined from linear equations: $s_{4}, c_{4}, \ldots, s_{6}, c_{6}$ from $T_{5} ; s_{3}, c_{3}, c_{7}$ from $K_{1} ; s_{1}, c_{1}, c_{2}$ from $K_{2}$.

For the number of singular configurations, note that we have second order equations for $s_{7}$, hence at most 2 values for the tuple $\left(s_{3}, c_{3}, s_{7}, c_{7}\right)$, and $s_{2}$. So in general if there are two separate roots both for $s_{7}$ and $s_{2}$, we get four different singularities.

Similar results can be presented for any $T_{i}$ but we will not catalogue them here.

\subsection{Subsystem 367}

Comparing to examples in Arp01 it was perhaps intuitively clear that subsystem $J_{4567}$ produces singularities. It is a bit more surprising that there is another subsystem producing singularities: the one formed by the nodes 3,6 , and 7 .

Define

$$
\begin{aligned}
h_{1} & :=-p_{5}+p_{1}=a_{6}\left(c_{6} c_{7}-s_{6} s_{7}\right)+a_{7} s_{7}-a_{3} s_{3}+w_{1}-b_{1} \\
h_{2} & :=-p_{6}+p_{2}=a_{6}\left(s_{6} c_{7}+c_{6} s_{7}\right)-a_{7} c_{7}+a_{3} c_{3}+w_{2}-b_{2} \\
h_{3} & :=p_{9}=c_{3}^{2}+s_{3}^{2}-1 \\
h_{4} & :=p_{12}=c_{6}^{2}+s_{6}^{2}-1 \\
h_{5} & :=p_{13}=c_{7}^{2}+s_{7}^{2}-1 .
\end{aligned}
$$


It is important to note that $h_{1}, h_{2}$ contain only angles 3,6 , and 7 , therefore only $p_{9}, p_{12}, p_{13}$ are relevant to them. As parameters we now have not only the lengths $a_{3}, a_{6}, a_{7}$, but also $b_{1}, \ldots, w_{2}$ i.e. the positions of the fixed nodes $A$ and $B$ in Figure 2. Let $J_{367}$ be the ideal generated by $h_{1}, \ldots, h_{5}$. We will proceed in a similar way as with the subsystem $J_{4567}$.

First we will consider the singularities of the subsystem $J_{367}$ using the following product order:

$$
J_{367} \cup F_{J_{367}} \subset \mathbb{Q}\left[\left(c_{3}, s_{3}, c_{6}, s_{6}, c_{7}, s_{7}\right),\left(a_{3}, a_{6}, a_{7}, b_{1}, b_{2}, w_{1}, w_{2}\right)\right]
$$

The relevant Gröbner basis $G$ contains 96 generators of which two are especially interesting:

$$
\begin{aligned}
& g_{12}=c_{6} a_{6} a_{7} \\
& g_{1}=\prod_{i=1}^{4} z_{i} \quad \text { where } \\
& z_{1}=\left(a_{3}-a_{6}+a_{7}\right)^{2}-|b-w|^{2} \\
& z_{2}=\left(a_{3}+a_{6}+a_{7}\right)^{2}-|b-w|^{2} \\
& z_{3}=\left(a_{3}+a_{6}-a_{7}\right)^{2}-|b-w|^{2} \\
& z_{4}=\left(a_{3}-a_{6}-a_{7}\right)^{2}-|b-w|^{2} .
\end{aligned}
$$

The latter one gives us the singular variety $S_{J_{367}}$.

Theorem 3. The singular variety of $J_{367}$ is

$$
S_{J_{367}}=\mathrm{V}\left(\left\langle g_{1}\right\rangle\right)
$$

Remark 4.4. It is worth noting that, contrary to the linear constraints $t_{i}$ in Theorem 1 related to $J_{4567}$, the $z_{i}$ in Theorem 3 give quadratic constraints $z_{i}=0$ related to $J_{367}$ and have the interpretation " $\left|a_{3} \pm a_{6} \pm a_{7}\right|=$ distance between the fixed points $\mathrm{A}$ and B". Furthermore, again the factors $z_{i}$ give the irreducible decomposition of the singular variety.

Since $a_{i}>0$, we get $c_{6}=0$ from $g_{12}=0$. This simplifies computations considerably. Let us define

$$
U:=\left\langle J_{367}, F_{J_{367}}, c_{6}\right\rangle
$$

The prime decomposition of $U$ turns out to have 8 components:

$$
\sqrt{U}=U_{1} \cap \cdots \cap U_{8}
$$

Inspecting the generators of each of $U_{i}$, it is noticed that the ideals $U_{k}, \quad k=5 \ldots 8$ contain generators which imply $a_{i}=0$ for some $i$. Hence those are discarded as non-physical and we are left with 4 ideals:

$$
\begin{aligned}
& U_{1}=\left\langle u_{1}, u_{2}, c_{7}^{2}+s_{7}^{2}-1, c_{6}, s_{6}-1, s_{3}+s_{7}, c_{3}+c_{7}\right\rangle \\
& U_{2}=\left\langle u_{1}, u_{2}, c_{7}^{2}+s_{7}^{2}-1, c_{6}, s_{6}+1, s_{3}+s_{7}, c_{3}+c_{7}\right\rangle \\
& U_{3}=\left\langle u_{1}, u_{2}, c_{7}^{2}+s_{7}^{2}-1, c_{6}, s_{6}+1, s_{3}-s_{7}, c_{3}-c_{7}\right\rangle \\
& U_{4}=\left\langle u_{1}, u_{2}, c_{7}^{2}+s_{7}^{2}-1, c_{6}, s_{6}-1, s_{3}-s_{7}, c_{3}-c_{7}\right\rangle \\
& \text { where }\left\{\begin{array}{l}
u_{1}=-s_{6} c_{7} a_{6}-c_{3} a_{3}+c_{7} a_{7}+b_{2}-w_{2} \\
u_{2}=s_{6} s_{7} a_{6}+s_{3} a_{3}-s_{7} a_{7}+b_{1}-w_{1} .
\end{array}\right.
\end{aligned}
$$


With these, we continue studying the whole system $J \cup F_{J}$. Each $U_{i}$ will lead to a different case with $s_{6}= \pm 1, s_{3}= \pm s_{7}, c_{3}= \pm c_{7}$. Let us look for example the ideal $U_{1} 4$ This gives

$$
\begin{aligned}
& s_{6}=1, \\
& c_{7}=\frac{b_{2}-w_{2}}{a_{6}-a_{3}-a_{7}}, \\
& s_{7}=\frac{b_{1}-w_{1}}{a_{3}-a_{6}+a_{7}}, \\
& c_{3}=-c_{7}, \\
& s_{3}=-s_{7} .
\end{aligned}
$$

We should expect to run into an equation $z_{i}=0$ for some $i$, where the expressions $z_{i}$ are given in (12). Combined with $c_{7}^{2}+s_{7}^{2}-1=0$ the equations (13) give $z_{1}=0$. Likewise, $U_{i}$ implies $z_{i}=0$ for $i=2,3,4$.

Remark 4.5. The condition $z_{2}=0$ is physically a redundant case: it means that the system can barely reach from $A$ to $B$ when the subsystem of the rods $a_{3}, a_{6}, a_{7}$ is fully stretched, i.e. it has no room to move. Therefore also $U_{2}$ corresponds to a rather trivial case. See also Remark 4.1.

Using $U_{1}$ we can now eliminate the variables corresponding to angles 3,6 , and 7 . Doing the substitutions in $J \cup F_{J}$ we are left with the following generators.

$$
\begin{aligned}
& L=\left\langle L_{1} \cup L_{2}\right\rangle, \\
& L_{1}:\left\{\begin{array}{l}
l_{1}=a_{2}\left(-c_{1} c_{2}+s_{1} s_{2}\right)+c_{1} a_{1}+s_{7} a_{3}-b_{1} \\
l_{2}=a_{2}\left(-s_{1} c_{2}-c_{1} s_{2}\right)+s_{1} a_{1}-c_{7} a_{3}-b_{2} \\
l_{3}=c_{1}^{2}+s_{1}^{2}-1 \\
l_{4}=c_{2}^{2}+s_{2}^{2}-1,
\end{array}\right. \\
& L_{2}:\left\{\begin{array}{l}
l_{5}=a_{4}\left(s_{4} c_{5}+c_{4} s_{5}\right)+c_{5} a_{5}+s_{7}\left(a_{6}-a_{7}\right) \\
l_{6}=a_{4}\left(c_{4} c_{5}-s_{4} s_{5}\right)-s_{5} a_{5}+c_{7}\left(a_{6}-a_{7}\right) \\
l_{7}=c_{4}^{2}+s_{4}^{2}-1 \\
l_{8}=c_{5}^{2}+s_{5}^{2}-1,
\end{array}\right.
\end{aligned}
$$

where the $s_{7}, c_{7}$ are no longer variables, but known expressions from (13) and kept here only for clarity of notation.

Remark 4.6. Before working on $L_{1}$ and $L_{2}$ we comment briefly on the other $U_{i}$ cases. Introduce $L_{3}$ and $L_{4}$ :

$$
\begin{aligned}
& L_{3}:\left\{\begin{array}{l}
a_{2}\left(-c_{1} c_{2}+s_{1} s_{2}\right)+c_{1} a_{1}-s_{7} a_{3}-b_{1}=0 \\
a_{2}\left(-s_{1} c_{2}-c_{1} s_{2}\right)+s_{1} a_{1}+c_{7} a_{3}-b_{2}=0 \\
c_{1}^{2}+s_{1}^{2}-1=0 \\
c_{2}^{2}+s_{2}^{2}-1=0
\end{array}\right. \\
& L_{4}:\left\{\begin{array}{l}
a_{4}\left(s_{4} c_{5}+c_{4} s_{5}\right)+c_{5} a_{5}-s_{7}\left(a_{6}+a_{7}\right)=0 \\
a_{4}\left(c_{4} c_{5}-s_{4} s_{5}\right)-s_{5} a_{5}-c_{7}\left(a_{6}+c_{7}\right)=0 \\
c_{4}^{2}+s_{4}^{2}-1=0 \\
c_{5}^{2}+s_{5}^{2}-1=0 .
\end{array}\right.
\end{aligned}
$$

${ }^{4}$ As with $J_{4567}$ and $T_{5}$, the other cases are completely similar and we will comment them shortly. 
Had we used $U_{2}$ instead of $U_{1}$, we would end up with the system $L_{1}, L_{4}$. Likewise, $U_{3}$ would give the system $L_{3}, L_{2}$, and $U_{4}$ would give the system $L_{3}, L_{4}$. Yet another point of view is, that $s_{6}= \pm 1$ picks between $L_{2}$ and $L_{4}$, while $\left(c_{3}, s_{3}\right)= \pm\left(c_{7}, s_{7}\right)$ picks between $L_{1}$ and $L_{3}$. More precisely, $s_{6}=1\left(s_{6}=-1\right)$ gives $L_{2}\left(L_{4}\right)$, and $\left(c_{3}, s_{3}\right)=\left(-c_{7},-s_{7}\right)$ gives $L_{1}$. The choice $\left(c_{3}, s_{3}\right)=\left(c_{7}, s_{7}\right)$ would give $L_{3}$.

Continuing with $L_{1}$ and $L_{2}$, we notice that $L_{2}$ contains only the variables $c_{5}, s_{5}, c_{4}, s_{4}$ (angles 4 and 5), has 4 equations and 4 variables hence is expected to have a finite solution set and will be handled analogously to the ideal $K_{2}$ in (6) . Calculating its Gröbner basis $G$ in the ring

$$
\mathbb{Q}\left(a_{4}, a_{5}, a_{6}, a_{7}\right)\left[\left(c_{4}, c_{5}, s_{5}, c_{7}, s_{7}\right),\left(s_{4}\right)\right]
$$

we obtain 12 generators, the first one being

$$
g_{1}=2 a_{4} a_{5} s_{4}+a_{4}^{2}+a_{5}^{2}-a_{6}^{2}+2 a_{6} a_{7}-a_{7}^{2} .
$$

Hence $s_{4}$ can be explicitly solved:

$$
s_{4}=\frac{a_{4}^{2}+a_{5}^{2}-a_{6}^{2}+2 a_{6} a_{7}-a_{7}^{2}}{-2 a_{4} a_{5}} .
$$

The other generators are too messy to be of much use. Then using the formula $c_{4}^{2}=1-s_{4}^{2}$ we get

$$
\begin{aligned}
c_{4}^{2} & =-\frac{\left(a_{4}+a_{5}-a_{6}+a_{7}\right)\left(a_{4}-a_{5}+a_{6}-a_{7}\right)\left(a_{4}-a_{5}-a_{6}+a_{7}\right)\left(a_{4}+a_{5}+a_{6}-a_{7}\right)}{4 a_{4}^{2} a_{5}^{2}} \\
& =-\frac{t_{7} t_{5} t_{6} t_{8}}{4 a_{4}^{2} a_{5}^{2}} .
\end{aligned}
$$

The product term in the numerator has to be nonpositive, in order to have any real solutions:

$$
t_{5} t_{6} t_{7} t_{8} \leq 0 \text {. }
$$

After solving $s_{4}, c_{4}$ we can proceed to solve $s_{5}$ and $c_{5}$. For this we use the ordering

$$
\mathbb{Q}\left(a_{4}, a_{5}, a_{6}, a_{7}\right)\left[c_{5}, s_{5}, c_{4}, s_{4}, c_{7}, s_{7}\right]
$$

and pick the two relevant equations from the corresponding Gröbner basis:

$$
\begin{aligned}
& \left(-a_{6}+a_{7}\right) s_{5}-a_{4} c_{4} s_{7}+a_{4} s_{4} c_{7}+a_{5} c_{7}=0 \\
& \left(-a_{6}+a_{7}\right) c_{5}-a_{4} c_{4} c_{7}-a_{4} s_{4} s_{7}-a_{5} s_{7}=0,
\end{aligned}
$$

which are linear equations for $s_{5}, c_{5}$, provided $a_{6} \neq a_{7}$.

Remark 4.7. In the case $a_{6}=a_{7}$ the situation is different: $L_{2}$ then decomposes into 3 prime ideals, of which only one is physically feasible and gives a singularity only if $a_{4}=a_{5}$. Thence this is a rather special case and will not be considered further here.

The subsystem $L_{2}$ is now fully solved. Moving on to $L_{1}$, we will see that the analysis is very similar to that of $K_{1}$ from ([6). Therefore we will skip some details. After forming the Gröbner basis of $L_{1}$ in the ring

$$
\mathbb{Q}\left(b_{1}, b_{2}, a_{1}, a_{2}, a_{3}, c_{7}, s_{7}\right)\left[c_{1}, s_{1}, c_{2}, s_{2}\right]
$$


with respect to the lexicographic ordering, we get for $s_{2}$, after simplifications, the relation

$$
\begin{aligned}
& s_{2}^{2}=\frac{n_{3}\left(4 a_{1} a_{2}-n_{3}\right)}{4 a_{1}^{2} a_{2}^{2}}, \\
& \quad \text { where } n_{3}=|b|^{2}+2 a_{3}\left(b_{2} c_{7}-b_{1} s_{7}\right)-\left(a_{1}-a_{2}\right)^{2}+a_{3}^{2}
\end{aligned}
$$

Again for the real solutions the numerator has to be nonnegative

$$
n_{3}\left(4 a_{1} a_{2}-n_{3}\right) \geq 0
$$

We can now solve $c_{2}, s_{1}$ and $c_{1}$, provided their coefficients are nonzero, from the linear equations

$$
\begin{aligned}
& 2 a_{1} a_{2} n_{4} c_{2}-4 a_{1}^{2} a_{2}^{2} s_{2}^{2}+r_{1}=0, \\
& -2 a_{1} n_{4} s_{1}+r_{2}+r_{3}=0, \\
& \left(a_{1}^{2}-a_{2}^{2}\right) c_{1}+r_{4}=0 .
\end{aligned}
$$

where

$$
n_{4}=|b|^{2}+a_{3}^{2}+2 a_{3}\left(b_{2} c_{7}-b_{1} s_{7}\right)
$$

and $r_{i}$ are lengthy, yet polynomial, expressions in the parameters, apart from $r_{4}$ which depends on $s_{1}, s_{2}, c_{2}$ as well. (See the appendix.)

What about the cases $n_{4}=0$ and/or $a_{1}=a_{2}$ ? It can be shown, as with $d_{0}$, that the condition $n_{3}\left(4 a_{1} a_{2}-n_{3}\right) \geq 0$ is equivalent to

$$
\left(a_{1}-a_{2}\right)^{2} \leq n_{4} \leq\left(a_{1}+a_{2}\right)^{2} .
$$

Therefore, if $a_{1} \neq a_{2}$ then $n_{4} \neq 0$ and the equations above are sufficient. The case $a_{1}=a_{2}$, $n_{4} \neq 0$ does not essentially change the situation: we still have a quadratic equation for $s_{2}$, and linear ones for the others, with a different coefficient for $c_{1}$.

The remaining case $a_{1}=a_{2}, n_{4}=0$ is analogous to the $n_{2}=0$ case within $J_{4567}$ and likewise will not be pursued further.

Theorem 4. Let us suppose that the parameters $a, b, w$ satisfy the following conditions:

$$
\begin{aligned}
& a_{6} \neq a_{7} \\
& n_{4} \neq 0 \\
& n_{3}\left(4 a_{1} a_{2}-n_{3}\right) \geq 0 \\
& t_{7} t_{5} t_{6} t_{8} \leq 0 .
\end{aligned}
$$

Then $V_{a, b}$ contains at least 2 singular points. If the inequalities are strict we get in general at least 4 singular points.

Similar results can be represented for any $\mathrm{V}\left(U_{i}\right)$ but we will not catalogue them here.

Proof. The last two conditions are due to univariate second degree polynomial equations, which have real solutions if and only if (201) (for $s_{2}$ ) and (21) (for $\left.c_{4}\right)$ are fulfilled. The first condition is needed for the other variables to be determined uniquely: $s_{3}, c_{3}, s_{6}, c_{6}, s_{7}, c_{7}$ from $\mathrm{V}\left(U_{1}\right), s_{4}, s_{5}, c_{5}$ from $L_{2}$, and $s_{1}, c_{1}, c_{2}$ from $L_{1}$.

For the number of singular configurations, note that we have second order equations, hence at most 2 values, for $c_{4}$ and $s_{2}$. So in general if there are two separate roots both for $c_{4}$ and $s_{2}$, we get four different singularities. 


\subsection{Two special cases with symmetry}

Let us look more closely at two special cases: $a_{4}=a_{6}, a_{5}=a_{7}$, and either $a_{4}=a_{5}$ or $a_{4} \neq a_{5}$.

\subsubsection{The case $a_{4} \neq a_{5}$}

Motivated by the original benchmark values [Sch90] we give the following

Lemma 4.1. When $a_{4}=a_{6}$ and $a_{5}=a_{7}$, there is a relation between the angles 4 and 6 : either $y_{6}=-y_{4}$ or $y_{6}=y_{4}+\pi$. Furthermore, if also $a_{4} \neq a_{5}$, the angle $y_{7}$ variables, i.e. $c_{7}, s_{7}$, are uniquely determined from $c_{4}, s_{4}, c_{5}, s_{5}$.

Proof. Looking for relations between solely angles 4 and 6 , we substitute $a_{4}=a_{6}$ and $a_{5}=a_{7}$ to the subsystem $J_{4567}$ and formulate a suitable elimination ideal. In idealtheoretic language, we define

$$
\begin{aligned}
& r_{1}:=a_{4}\left(s_{4} c_{5}+c_{4} s_{5}\right)+a_{5} c_{5}-a_{4}\left(c_{6} c_{7}-s_{6} s_{7}\right)-a_{5} s_{7} \\
& r_{2}:=a_{4}\left(c_{4} c_{5}-s_{4} s_{5}\right)-a_{5} s_{5}+a_{4}\left(s_{6} c_{7}+c_{6} s_{7}\right)-a_{5} c_{7} \\
& r_{i+2}=c_{i+3}^{2}+s_{i+3}^{2}-1, \quad i=1, \ldots, 4
\end{aligned}
$$

where $r_{i}=q_{i}$ with substitutions $a_{4}=a_{6}$ and $a_{5}=a_{7}$, and investigate the ideal $I:=$ $\left\langle r_{1}, \ldots, r_{6}\right\rangle$ in the ring

$$
\mathbb{Q}\left(a_{4}, a_{5}, a_{6}, a_{7}\right)\left[\left(c_{5}, s_{5}, c_{7}, s_{7}\right),\left(c_{4}, s_{4}, c_{6}, s_{6}\right)\right] .
$$

Calculating the elimination ideal $I_{4,6}:=I \cap \mathbb{Q}\left[c_{4}, s_{4}, c_{6}, s_{6}\right]$ we get

$$
I_{4,6}=\left\langle s_{4}+s_{6}, c_{6}^{2}+s_{6}^{2}-1, c_{4}^{2}+s_{4}^{2}-1\right\rangle .
$$

Calculating the prime decomposition of $\sqrt{I_{4,6}}$ we get

$$
\sqrt{I_{4,6}}=\left\langle c_{6}^{2}+s_{6}^{2}-1, c_{4}-c_{6}, s_{4}+s_{6}\right\rangle \cap\left\langle c_{6}^{2}+s_{6}^{2}-1, c_{4}+c_{6}, s_{4}+s_{6}\right\rangle .
$$

Since $I_{4,6} \subset I \subset J \subset J \cup F_{J}$, we have

$$
\mathrm{V}\left(I_{4,6}\right) \supset \mathrm{V}\left(J \cup F_{J}\right)
$$

From these prime ideals we can see that everywhere in $\mathrm{V}\left(I_{4,6}\right)$, and therefore in the variety of the singularities of the whole system as well, $s_{6}=-s_{4}$ and either $c_{6}=c_{4}$ or $c_{6}=-c_{4}$. These translate into two possible relations between the angles $y_{4}$ and $y_{6}$.

$$
\left(c_{6}, s_{6}\right)=\left(c_{4},-s_{4}\right) \Leftrightarrow y_{6}=-y_{4}, \quad\left(c_{6}, s_{6}\right)=\left(-c_{4},-s_{4}\right) \Leftrightarrow y_{6}=y_{4}+\pi .
$$

This proves the first claim. If we take into account either one of the prime ideals of $\sqrt{I_{4,6}}$ in $I$ and calculate the Gröbner bases we get ideals where $c_{7}$ and $s_{7}$ depend linearly on $c_{4}$, $s_{4}, c_{5}$ and $s_{5}$, and can be explicitely solved, as we will show next to prove the latter claim of the lemma. For the case $\left(s_{6}, c_{6}\right)=\left(-s_{4},-c_{4}\right)$ we get

$$
\left\{\begin{array}{l}
c_{7}=-s_{5} \\
s_{7}=c_{5}
\end{array} \text { which imply } y_{7}=y_{5}+\frac{\pi}{2} .\right.
$$


For the case $\left(s_{6}, c_{6}\right)=\left(-s_{4}, c_{4}\right)$ the expressions are, albeit linear, slightly more complicated:

$$
\begin{aligned}
& c_{7}\left(a_{4}^{2}\left(s_{4}^{2}-c_{4}^{2}\right)-a_{5}\left(2 a_{4} s_{4}+a_{5}\right)\right)+s_{7}\left(2 a_{4}\left(a_{5}+a_{4} c_{4} s_{4}\right)\right)-s_{5}\left(\left(a_{4}^{2}+a_{5}^{2}\right)-2 a_{4} a_{5} s_{4}\right)=0 \\
- & \left.c_{7}\left(2 a_{4}^{2} c_{4} s_{4}\right)+s_{7}\left(a_{4}^{2}\left(c_{4}^{2}-s_{4}^{2}\right)+a_{5}^{2}\right)\right)+\left(a_{4}^{2}-a_{5}^{2}\right) c_{5}-2 a_{4} a_{5} s_{5} c_{4}=0 .
\end{aligned}
$$

We prove that these indeed determine $c_{7}, s_{7}$ : all we need to do is check that the determinant of the coefficient matrix $A$ of the linear equations does not equal zero:

$$
A:=\left(\begin{array}{cc}
a_{4}^{2}\left(s_{4}^{2}-c_{4}^{2}\right)-a_{5}\left(2 a_{4} s_{4}+a_{5}\right) & 2 a_{4}\left(a_{5}+a_{4} c_{4} s_{4}\right) \\
-2 a_{4}^{2} c_{4} s_{4} & a_{4}^{2}\left(c_{4}^{2}-s_{4}^{2}\right)+a_{5}^{2}
\end{array}\right), \text { prove } \operatorname{det}(A) \neq 0 .
$$

Now $\operatorname{det}(A)$ simplifies due to $c_{4}^{2}+s_{4}^{2}=1$, resulting in

$$
\operatorname{det}(A)=2 a_{4} a_{5}\left(a_{4}+a_{5}\right)\left(a_{4}-a_{5}\right) s_{4}+\left(a_{4}-a_{5}\right)\left(a_{4}+a_{5}\right)\left(a_{4}^{2}+a_{5}^{2}\right)
$$

Let us then consider $\operatorname{det}(A)$ as a function of $s_{4}$. Since $s_{4} \in[-1,1], \operatorname{det}(A):[-1,1] \mapsto \mathbb{R}$. Clearly if $a_{4}=a_{5}, \operatorname{det}(A) \equiv 0$ so we need to assume $a_{4} \neq a_{5}$. Set

$$
h\left(s_{4}\right):=\frac{\operatorname{det}(A)}{\left(a_{4}+a_{5}\right)\left(a_{4}-a_{5}\right)}=2 a_{4} a_{5} s_{4}+\left(a_{4}^{2}+a_{5}^{2}\right)
$$

and inspect when $h=0$. Since $a_{4}>0$ and $a_{5}>0$ the linear function $h$ has its minimum at -1 .

$$
h(-1)=a_{4}^{2}+a_{5}^{2}-2 a_{4} a_{5}=\left(a_{5}-a_{4}\right)^{2}>0 .
$$

This proves $h \neq 0$ always, therefore under the assumption $a_{4} \neq a_{5}$ also $\operatorname{det}(A) \neq 0$ as claimed.

\subsubsection{The case $a_{4}=a_{5}$}

We study the special case $a_{4}=a_{5}=a_{6}=a_{7}$, whence the 4567-subsystem is capable of "buckling" in more complicated ways, thereby producing further interesting configurations. This resembles then the net example in Arp01.

Let us see how $J_{4567}$ simplifies with substitutions $a_{4}=a_{5}=a_{6}=a_{7}$. Note that the assumptions of Lemma 4.1 considering $y_{7}$ are no longer valid. Let

$$
I:=J_{4567} \text { with } a_{4}=a_{5}=a_{6}=a_{7} \text { and } s_{6}=-s_{4}
$$

and compute its prime decomposition. This results in

$$
\begin{gathered}
\sqrt{I}=I_{1} \cap I_{2} \cap I_{3} \quad \text { with generators } \\
I_{1}=\left\{\begin{array}{l}
s_{4}^{2}+c_{6}^{2}-1, \\
c_{4}-c_{6}, \\
c_{7}^{2}+s_{7}^{2}-1, \\
s_{5}+c_{7} s_{4}-s_{7} c_{6}, \\
c_{5}-c_{7} c_{6}-s_{7} s_{4}
\end{array} \quad I_{2}=\left\{\begin{array}{l}
c_{6}, \\
s_{4}+1, \\
c_{4}, \\
c_{7}^{2}+s_{7}^{2}-1, \\
c_{5}^{2}+s_{5}^{2}-1
\end{array} \quad I_{3}=\left\{\begin{array}{l}
s_{4}^{2}+c_{6}^{2}-1, \\
c_{4}+c_{6}, \\
c_{7}^{2}+s_{7}^{2}-1, \\
s_{5}+c_{7}, \\
c_{5}-s_{7}
\end{array}\right.\right.\right.
\end{gathered}
$$

Each of these has a geometrical interpretation, see Figure 3. $I_{2}$ corresponds to $y_{4}=$ $-\pi / 2, y_{6}=\pi / 2$ which means that nodes $A$ and $P_{2}$ coincide. This is like the $T_{5}$ situation. Indeed, the ideal $J \cup F_{J} \cup I_{2}$ turns out to be exactly $T_{5}$ with the extra condition $a_{4}=a_{5}$. 
Although it is not immediately apparent but in that situation there also arises a new condition among the parameters: $a_{3}=|b-w|$, i.e. " $a_{3}$ equals the distance between $A$ and $B$ ". Note that here the Fitting ideal $F_{J_{4567}}$ has not been used at all, contrary to the $T_{5}$ calculations.

$I_{3}$ corresponds to $y_{6}=y_{4}+\pi$ and $y_{5}=y_{7}-\pi / 2$ so that now nodes $P_{3}$ and $P_{4}$ coincide. Then again, $I_{1}$ corresponds to $y_{6}=-y_{4}$ and $y_{5}=y_{6}+y_{7}$, which interestingly is not a singularity but merely expressing a symmetry in the system due to $a_{4}=a_{5}=a_{6}=a_{7}$.

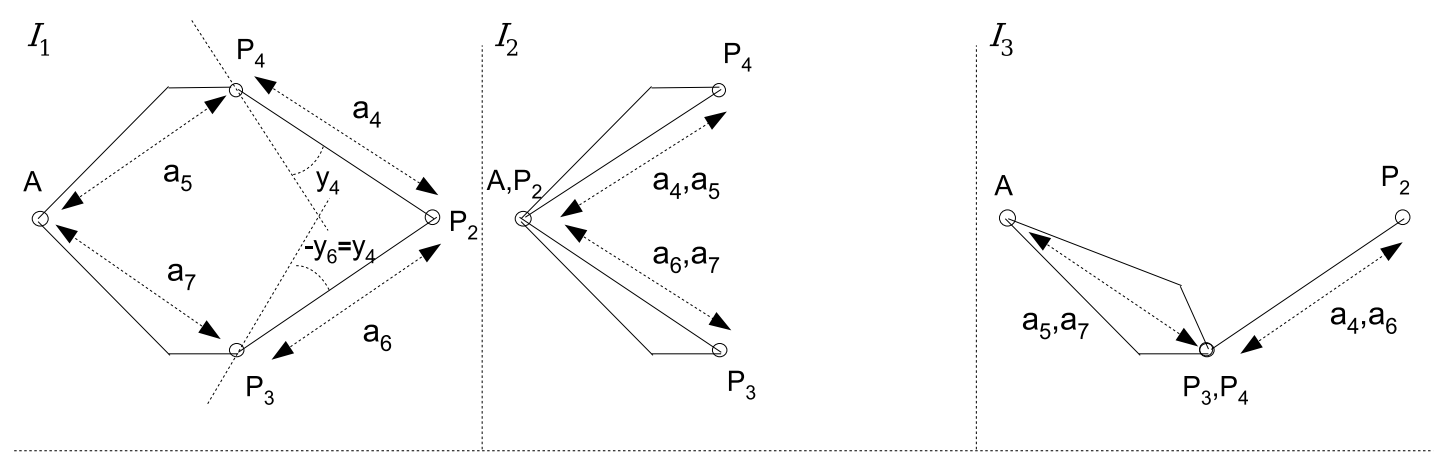

Figure 3: The configurations corresponding to $I_{1}, I_{2}, I_{3}$ in the case $a_{4}=a_{5}=a_{6}=a_{7}$.

\subsection{Other subsystems}

Now contemplating Figure 2 we see that it would be possible to find other singularities by analysing still other subsystems. For example the subsystem corresponding to rods 3,4 and 5 is by symmetry similar to subsystem 367: we simply exchange the roles of variables and parameters associated to rods 4 and 6 , and 5 and 7 . Further we could consider other subsystems formed from different "paths" between the nodes $A, B, O$ : i.e. subsystems $J_{123}, J_{1245}, J_{1267}$. Again by symmetry the system $J_{1267}$ is completely similar to $J_{1245}$, but cases $J_{123}$ and $J_{1245}$ give new singularities. We checked that in these cases the singular variety is not empty, and that at least for some parameter values we get singular points.

We did not analyse these cases in detail because computations are quite similar to those given above for subsystems $J_{4567}$ and $J_{367}$. Hence we did not feel including these would give significant additional value and therefore left them out to avoid expanding this quite a long presentation further.

\section{Numerical examples}

In this section we will calculate numerical examples for both types of singularities. Interestingly, the explicit expressions within $\tilde{G}, \hat{G}$, as well as in the Gröbner bases of $L_{1}$ and $L_{2}$, are unstable for numerical computations. It is better to use the original defining equations of $K_{1}, K_{2}, L_{1}, L_{2}$ in the computations. We shall not explore this stability issue here due to its non-relevance for the present context.

We present 4 examples:

1. The original benchmark parameter values, see [MI03]. We show that then the system is avoiding singularities 5

\footnotetext{
${ }^{5}$ Thereby validating its benchmark status. That is, the numerical difficulties encountered there are indeed due to the "numerical stiffness" of the problem, not to a nearby singularity.
} 
2. We explore how should $a_{1}, a_{2}$ be changed in order to have $J_{4567}$ type singularities in the system. Here we have an interpretation for the result: the lengths $a_{1}, a_{2}$ must be such that the subsystem 4567 has room for a certain kind of "buckled" configuration.

3. We explore how should $b_{1}, a_{1}, a_{2}$ be changed in order to have $J_{367}$ type singularities in the system.

4. A special case which shows a rational solution, that is $c_{i}, s_{i} \in \mathbb{Q}$ for all $i$. This shows unambiguously that we can find singular points because in this case there are no numerical errors related to floating point computations.

\subsection{Original values}

In this example, we will use the original values for the parameters $a_{i}, b_{i}$ and show that the system then has no singularities. The original parameters used in the benchmark tests [Sch90, HW91, MI03] are

$$
\begin{aligned}
& a_{1}=0.007 \quad a_{2}=0.028 \quad a_{3}=0.035 \quad a_{4}=0.020 \quad a_{5}=0.040 \quad a_{6}=0.020 \quad a_{7}=0.040 \\
& b_{1}=-0.03635 \quad b_{2}=0.03273 \quad w_{1}=-0.06934 \quad w_{2}=-0.00227
\end{aligned}
$$

Since $a_{7}=a_{5}$ and $a_{6}=a_{4}$, we have $t_{4}=t_{6}=0$ (and $t_{1}<0, t_{5}<0$ ) so we could have an $J_{4567}$ singularity: $T_{3}$ or $T_{5}$.

Remark 5.1. Interpretation: both $T_{3}$ and $T_{5}$ describe a situation where the 4567 system has 'collapsed' into a 1-dimensional object. The ideal $K_{2}$ tells us how $a_{3}$ restricts the possible attitudes of 4567 . In $T_{5}$ the centre node $P_{2}$ has been pushed in, in $T_{3}$ it has been pulled out.

Let us look more closely first at $T_{5}$, say, and check the conditions (8) and (10). The first one is fulfilled. For $E$ we first need to solve $c_{3}, s_{3}$ from $\mathrm{V}\left(K_{2}\right)$. Their solutions are

$$
\begin{array}{r}
\left(c_{3}, s_{3}, c_{7}, s_{7}\right) \in\{(0.4299535996,-0.9028509856,-0.9975812008,0.06951077517), \\
(0.9266735994,-0.3758670513,-0.1283212011,0.9917326602)\}
\end{array}
$$

With these $c_{3}, s_{3}$ we can compute $E$. Both sets in (26) give $E=\mathcal{O}\left(10^{-5}\right)>0$ and the condition (10) is violated, hence there are no $\left(J_{4567}-\right)$ singularities. What about other singularities? This is answered by the following

Theorem 5. With the original benchmark parameter values (25), the Andrews' squeezing system has no singularities.

Proof. We now have $a_{4}=a_{6}, a_{5}=a_{7}$ and $a_{4} \neq a_{5}$. Lemma 4.1 implies variables $c_{6}, s_{6}$, $c_{7}, s_{7}$, and so $y_{6}$ and $y_{7}$ can be explicitely solved in terms of $c_{4}, s_{4}, c_{5}$, and $s_{5}$. It is then possible to reduce the original system of constraint equations, by forgetting the last two equations from (2), and consider

$$
\begin{cases}a_{1} \cos \left(y_{1}\right)-a_{2} \cos \left(y_{1}+y_{2}\right)-a_{3} \sin \left(y_{3}\right)-b_{1} & =0 \\ a_{1} \sin \left(y_{1}\right)-a_{2} \sin \left(y_{1}+y_{2}\right)+a_{3} \cos \left(y_{3}\right)-b_{2} & =0 \\ a_{1} \cos \left(y_{1}\right)-a_{2} \cos \left(y_{1}+y_{2}\right)-a_{4} \sin \left(y_{4}+y_{5}\right)-a_{5} \cos \left(y_{5}\right)-w_{1} & =0 \\ a_{1} \sin \left(y_{1}\right)-a_{2} \sin \left(y_{1}+y_{2}\right)+a_{4} \cos \left(y_{4}+y_{5}\right)-a_{5} \sin \left(y_{5}\right)-w_{2} & =0\end{cases}
$$


These are equivalent to

$$
\begin{cases}a_{1} \cos \left(y_{1}\right)-a_{2} \cos \left(y_{1}+y_{2}\right)-a_{3} \sin \left(y_{3}\right)-b_{1} & =0 \\ a_{1} \sin \left(y_{1}\right)-a_{2} \sin \left(y_{1}+y_{2}\right)+a_{3} \cos \left(y_{3}\right)-b_{2} & =0 \\ -a_{4} \sin \left(y_{4}+y_{5}\right)-a_{5} \cos \left(y_{5}\right)+a_{3} \sin \left(y_{3}\right)+\left(b_{1}-w_{1}\right) & =0 \\ a_{4} \cos \left(y_{4}+y_{5}\right)-a_{5} \sin \left(y_{5}\right)-a_{3} \cos \left(y_{3}\right)+\left(b_{2}-w_{2}\right) & =0\end{cases}
$$

These can be again represented as polynomials.

$$
\begin{aligned}
& m_{1}:=a_{1} c_{1}-a_{2}\left(c_{1} c_{2}-s_{1} s_{2}\right)-a_{3} s_{3}-b_{1}=0 \\
& m_{2}:=a_{1} s_{1}-a_{2}\left(s_{1} c_{2}+c_{1} s_{2}\right)+a_{3} c_{3}-b_{2}=0 \\
& m_{3}:=a_{1} c_{1}-a_{2}\left(c_{1} c_{2}-s_{1} s_{2}\right)-a_{4}\left(s_{4} c_{5}+c_{4} s_{5}\right)-a_{5} c_{5}-w_{1}=0 \\
& m_{4}:=a_{1} s_{1}-a_{2}\left(s_{1} c_{2}+c_{1} s_{2}\right)+a_{4}\left(c_{4} c_{5}-s_{4} s_{5}\right)-a_{5} s_{5}-w_{2}=0 \\
& m_{i+4}:=c_{i}^{2}+s_{i}^{2}-1=0, \quad i=1, \ldots, 5 .
\end{aligned}
$$

Substituting the original parameter values (25), as rational numbers, into the polynomials $m_{i}$ we form an ideal $I:=\left\langle m_{1}, \ldots, m_{9}\right\rangle$. Let $K:=I \cup F_{I}$, where $F_{I}$ is the Fitting ideal of $I$, and inspect $K$ in the ring

$$
\mathbb{Q}\left[\left(c_{1}, s_{1}, c_{2}, s_{2}\right),\left(c_{3}, s_{3}, c_{4}, s_{4}, c_{5}, s_{5}\right)\right] .
$$

Now it is possible to compute the Gröbner basis $G_{K}$ for $K$ explicitly (unlike for $J \cup F_{J}$ in the introduction) and results in

$$
G_{K}=\langle 1\rangle
$$

This implies $\mathrm{V}(K)=\emptyset$, proving that with these original parameter values there are no singularities.

\section{$5.2 J_{4567}$ singularity: original values, apart from $a_{1}, a_{2}$}

Let us see how changing $a_{1}$ and/or $a_{2}$ might produce $J_{4567}$ type singularities. Our analysis reveals that by suitable combinations of $a_{1}$ and $a_{2}$ we can get between zero and four singularities (of type $J_{4567}$, that is). The number of singularities is determined by $c_{3}, s_{3}$, and $E$.

Considering $E$ as a function of $a_{1}, a_{2}$ we plot the area where $E \leq 0$. Recall that $E$ depends on $c_{3}$ as well, and $c_{3}$ has two possible values so we get two functions: $E=$ $E_{1}\left(a_{1}, a_{2}\right)$ (resp. $E=E_{2}\left(a_{1}, a_{2}\right)$ ) corresponding to the first (resp. second) value of $c_{3}$ from (26). See Figure 4 where the areas inside the rectangular areas are $E_{i}<0$.

- no singularities: $E_{1}>0, E_{2}>0$.

- 1 singularity: $E_{1}=0, E_{2}=0$, which leads (with $T_{5}$ ) to two possible values:

$$
\left(a_{1}=0.05986, a_{2}=0.01035\right), \quad\left(a_{1}=0.01035, a_{2}=0.05986\right)
$$

- 2 singularities: one of $E_{1}, E_{2}$ is $<0$, the other one $>0$.

- 3 singularities: one of $E_{1}, E_{2}$ is $<0$, the other one $=0$.

- 4 singularities: $E_{1}<0, E_{2}<0$. 

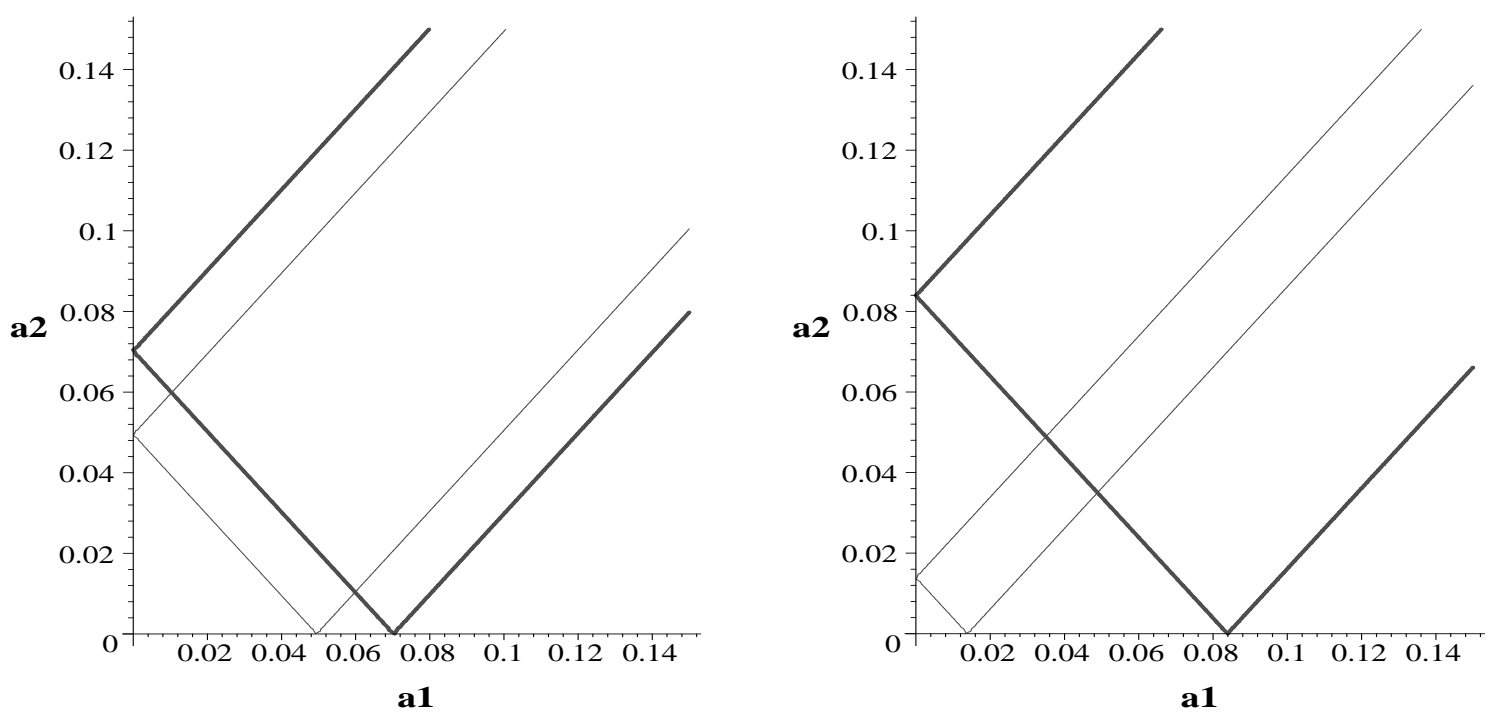

Figure 4: The rectangular lines are $E_{1}=0$ (thick line) and $E_{2}=0$ (thin line), the areas inside each $E_{i}=0$ line are where $E_{i}<0$. Left panel: $T_{5}$ case, right panel: $T_{3}$ case.

For example, let us concentrate on $T_{5}$ and choose $a_{1}=0.03, a_{2}=0.055$, say, whence the system is able to reach four singular configurations (see the left panel of Figure 4). Now $s_{i}, c_{i}$ for $i=1,2,3,7$ are determined by $\mathrm{V}(K)$. The other values, for angles 4,5,6, are determined by $\mathrm{V}\left(T_{5}\right)$. The results are in the Table 1. The corresponding configurations are visualized in Figure 5. Doing similar tests with $T_{3}$ instead of $T_{5}$ yields the $E_{i}$ areas in the right hand panel of Figure 4. Singular configurations implied by $T_{3}$, with choices $a_{1}=0.06, a_{2}=0.06$ which imply 4 singularities, are in Figure 6. To save space we have not tabulated the actual values of the angles in $T_{3}$ case.

\section{$5.3 J_{367}$ singularity: original values, apart from $b_{1}, a_{1}, a_{2}$}

A necessary condition to have a $J_{367}$ type singularity is at least one of the $z_{i}$ 's vanishes (12). Substituting the original parameter values we notice that none of these is zero. Let us then investigate how we should change some of the parameters in order to have $J_{367}$ type singularities. Take $b_{1}$ and $U_{1}$, say, and choose $b_{1}:=-0.026913593$ so that $z_{1}=0$. 6 We seek to further fulfil the sufficient requirements by $U_{1}$ :

$$
\begin{aligned}
& n_{3}\left(4 a_{1} a_{2}-n_{3}\right) \geq 0 \\
& t_{7} t_{5} t_{6} t_{8} \leq 0,
\end{aligned}
$$

and use $L_{1}, L_{2}$ to find the actual singular configurations. With the original parameter values $t_{6}=0$, therefore (21) is fulfilled. Therefore we only need to study (201). For that, we proceed analogously to Example 5.2 : treat the expression $n_{3}\left(4 a_{1} a_{2}-n_{3}\right)$ as a function of $a_{1}, a_{2}$. For that, we first need $c_{7}, s_{7}$. Them we get from (13)

$$
\begin{aligned}
& c_{7}=\frac{b_{2}-w_{2}}{a_{6}-a_{3}-a_{7}}=-0.6364 \\
& s_{7}=\frac{b_{1}-w_{1}}{a_{3}+a_{7}-a_{6}}=0.7714 .
\end{aligned}
$$

The region of $a_{1}, a_{2}$ plane where $n_{3}\left(4 a_{1} a_{2}-n_{3}\right) \geq 0$ is shown in Figure 7 . We pick a value

\footnotetext{
${ }^{6}$ This corresponds to moving $B$ slightly to left.
} 


\begin{tabular}{|c|c|c|c|c|}
\hline variable & singularity 1 & singularity 2 & singularity 3 & singularity 4 \\
\hline$c_{1}$ & -0.8322 & -0.4564 & -0.1157 & -0.1038 \\
\hline$s_{1}$ & -0.5544 & 0.8898 & -0.9933 & 0.9946 \\
\hline$c_{2}$ & -0.3045 & -0.3045 & 0.4467 & 0.4467 \\
\hline$s_{2}$ & 0.9525 & -0.9525 & 0.8947 & -0.8947 \\
\hline$c_{3}$ & 0.4300 & 0.4300 & 0.9267 & 0.9267 \\
\hline$s_{3}$ & -0.9029 & -0.9029 & -0.3759 & -0.3759 \\
\hline$c_{4}$ & 0 & 0 & 0 & 0 \\
\hline$s_{4}$ & -1 & -1 & -1 & -1 \\
\hline$c_{5}$ & 0.0695 & 0.0695 & 0.9917 & 0.9917 \\
\hline$s_{5}$ & 0.9976 & 0.9976 & 0.1283 & 0.1283 \\
\hline$c_{6}$ & 0 & 0 & 0 & 0 \\
\hline$s_{6}$ & 1 & 1 & 1 & 1 \\
\hline$c_{7}$ & -0.9976 & -0.9976 & -0.1283 & -0.1283 \\
\hline$s_{7}$ & 0.0695 & 0.0695 & 0.9917 & 0.9917 \\
\hline
\end{tabular}

Calculating the corresponding angles we get the following values.

\begin{tabular}{|c|c|c|c|c|}
\hline Angle & singularity 1 & singularity 2 & singularity 3 & singularity 4 \\
\hline$y_{1}$ & -2.5539 & 2.0448 & -1.6867 & 1.6747 \\
\hline$y_{2}$ & 1.8802 & -1.8802 & 1.1077 & -1.1077 \\
\hline$y_{3}$ & -1.1264 & -1.1264 & -0.3853 & -0.3853 \\
\hline$y_{4}$ & -1.5708 & -1.5708 & -1.5708 & -1.5708 \\
\hline$y_{5}$ & 1.5012 & 1.5012 & 0.1287 & 0.1287 \\
\hline$y_{6}$ & 1.5708 & 1.5708 & 1.5708 & 1.5708 \\
\hline$y_{7}$ & 3.0720 & 3.0720 & 1.6995 & 1.6995 \\
\hline
\end{tabular}

Table 1: The singularities of $J_{4567}$ type, original values apart from $a_{1}, a_{2}$. The values are presented only with 4 decimals but were computed with 16 decimals.

inside the "allowed" annulus, say $a_{1}=0.02$ and $a_{2}=0.055$ in order to get singularities. Then let us find the actual singular configurations: since $t_{6}=0$, from (16) we get $c_{4}=0$ and from (15) $s_{4}=-1$. The other angles are found as follows: 3 and 6 from (13) and the remaining ones 1,2,5 from $L$. The results are in Table 2, The corresponding singular configurations are drawn in Figure 8. Note that there are only two singular configurations, instead of four, since (16) has only one (double) root $c_{4}=0$ instead of two separate roots.

\subsection{A rational case}

Finally, let us show a rational valued singularity, that is $c_{i}, s_{i} \in \mathbb{Q}$. Choose

$$
\begin{array}{cccc}
a_{4}=a_{5}=a_{6}=a_{7}=3 / 20 & a_{1}=1 / 10 & a_{2}=a_{3}=1 / 2 \\
b_{1}=-1 / 10 & b_{2}=1 / 5 & w_{1}=-2 / 5 & w_{2}=-1 / 5
\end{array}
$$




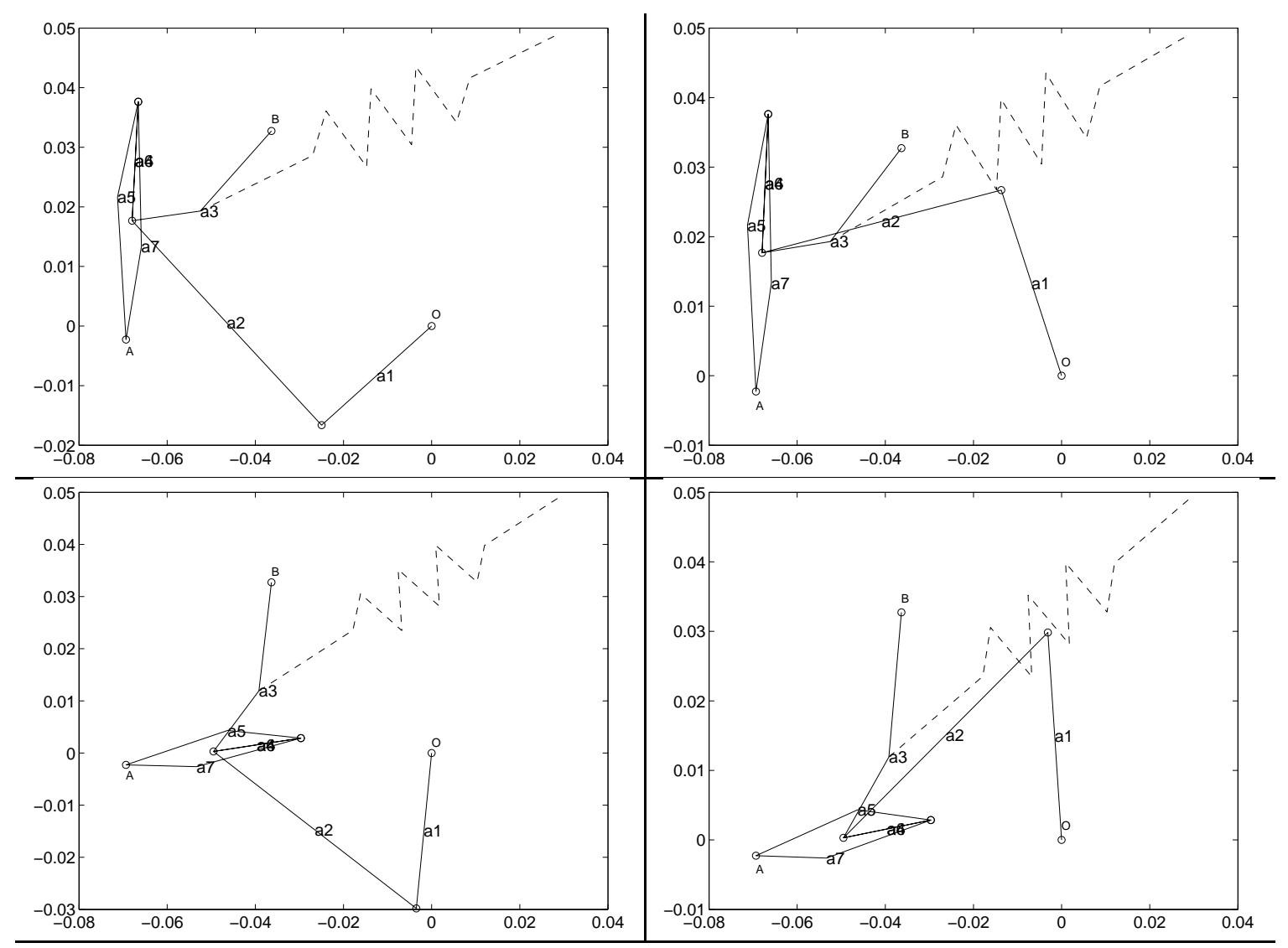

Figure 5: Singular positions (according to $J_{4567}, T_{5}$ ) when $a_{1}=0.03, a_{2}=0.055$ and $a_{3}, \ldots, a_{7}$ have the original values. One can see a physical explanation to the singularity: the centre node $P_{2}$ is 'pushed in' so that nodes $P_{3}$ and $P_{4}$ coincide.

and solve $c, s$ from the generators of $I_{2} \cup J \cup F_{J}$ in (24). Now $c_{5}, s_{5}, c_{7}, s_{7}$ are arbitrary (apart from $c_{5}^{2}+s_{5}^{2}=1, c_{7}^{2}+s_{7}^{2}=1$ ) and the chosen result is (see also Figure 9)

$$
\begin{aligned}
& c=(0,3 / 5,4 / 5,0,3 / 5,0,4 / 5) \\
& s=(1,-4 / 5,-3 / 5,-1,4 / 5,1,3 / 5) .
\end{aligned}
$$

\section{Conclusion}

We have studied singularities of the multibody system "Andrews' squeezing system" which is a well-known benchmark problem both for multibody solvers and differential-algebraic equation solvers. Using our tools we have shown in Theorem 5 that the original benchmark problem is indeed void of singularities, thereby assuring that whatever numerical problems in the benchmark tests are met, they are indeed due to something else than a nearby singularity of the system. Apparently, this non-singularity of the problem has not been rigorously proven in the literature.

However, we have shown that with suitably chosen parameters $(a, b, w)$, this system can exhibit singular configurations. In fact, there are families of values $(a, b, w)$ that produce singularities, see Theorems 2 and 4 . We provide examples of singularities, calculated using the original benchmark parameter values apart from $b_{1}, a_{1}, a_{2}$. Considering $a_{1}, a_{2}$ as freely chosen parameters, Figures 4 and 7 show the areas of $a_{1}, a_{2}$ plane where the system 


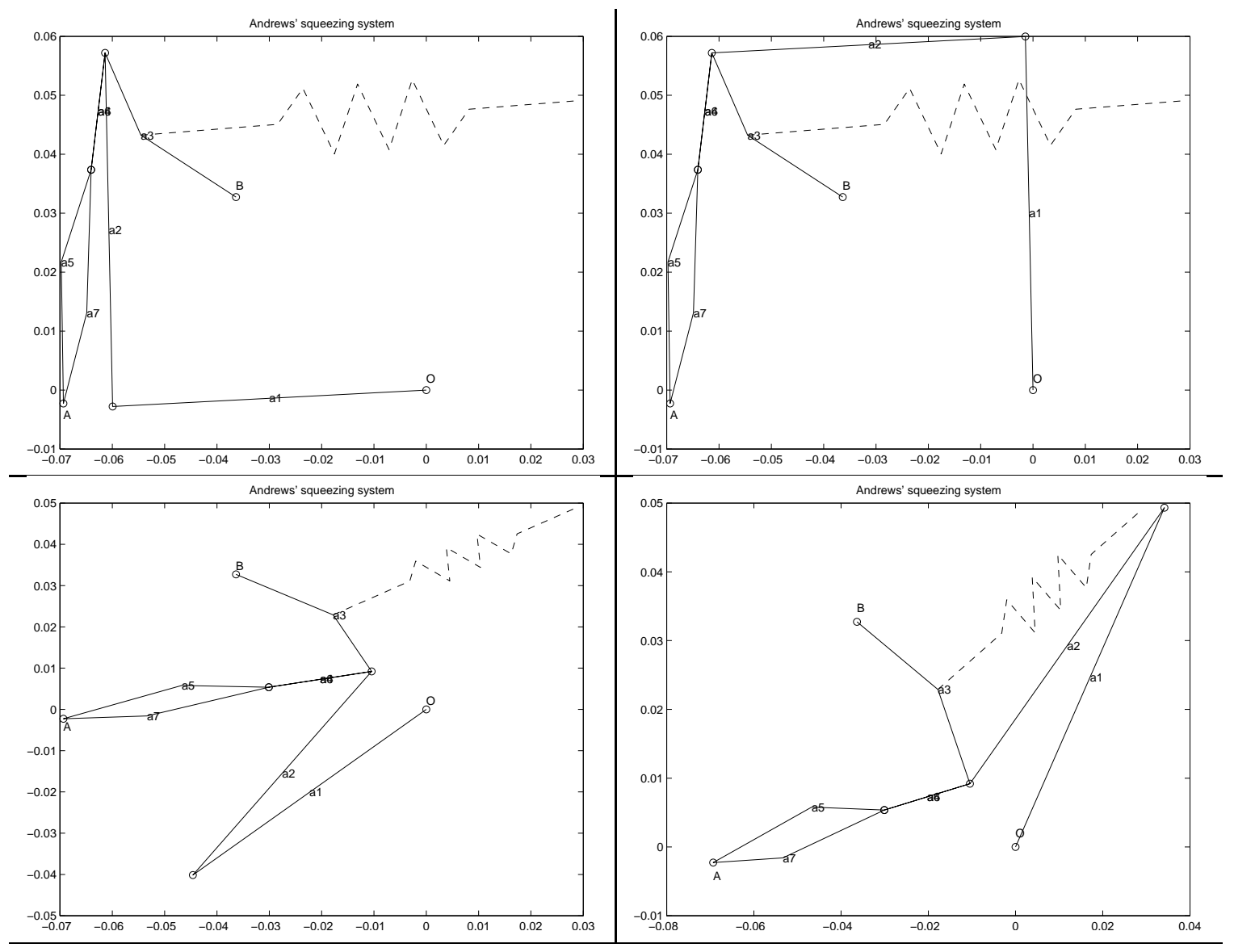

Figure 6: Singular positions (according to $J_{4567}, T_{3}$ ) when $a_{1}=0.06, a_{2}=0.06$ and $a_{3}, \ldots, a_{7}$ have the original values. One can see a physical explanation to the singularity: the centre node $P_{2}$ is now 'pulled out' so that nodes $P_{3}$ and $P_{4}$ coincide.

exhibits singularities. For example, choosing the point $\left(a_{1}, a_{2}\right)$ within the intersection of the three areas in Figures 4 (both panels) and 7 would give a system with 10 singular configurations.

A natural question that remains is, if these presented singularities are the only possible ones? In other words are there singularities which do not come from the singularities of some subsystem? While the Gröbner bases techniques in principle provide a way to answer this question directly, we could not do so in practice due to complexity problems. 


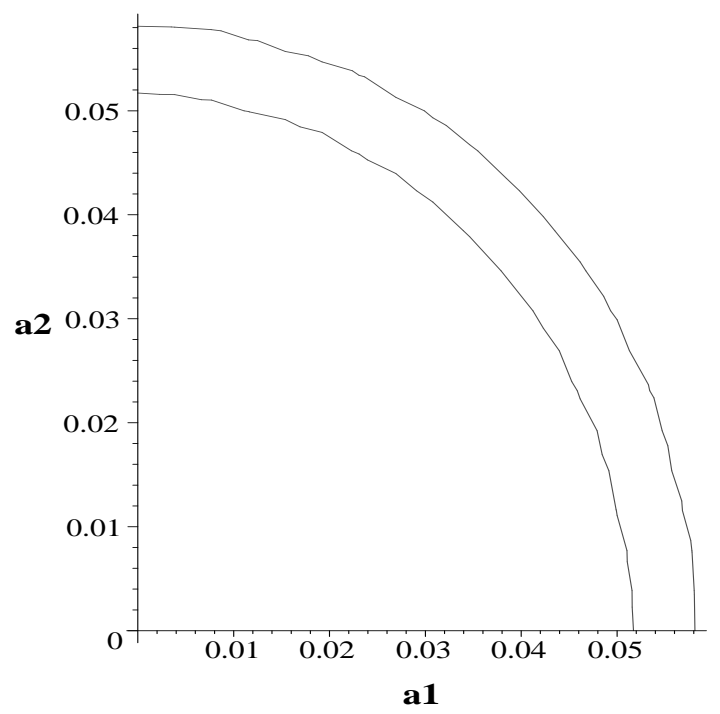

Figure 7: $J_{367}, U_{1}$ case: the region inside the annulus is where $n_{3}\left(4 a_{1} a_{2}-n_{3}\right) \geq 0$.
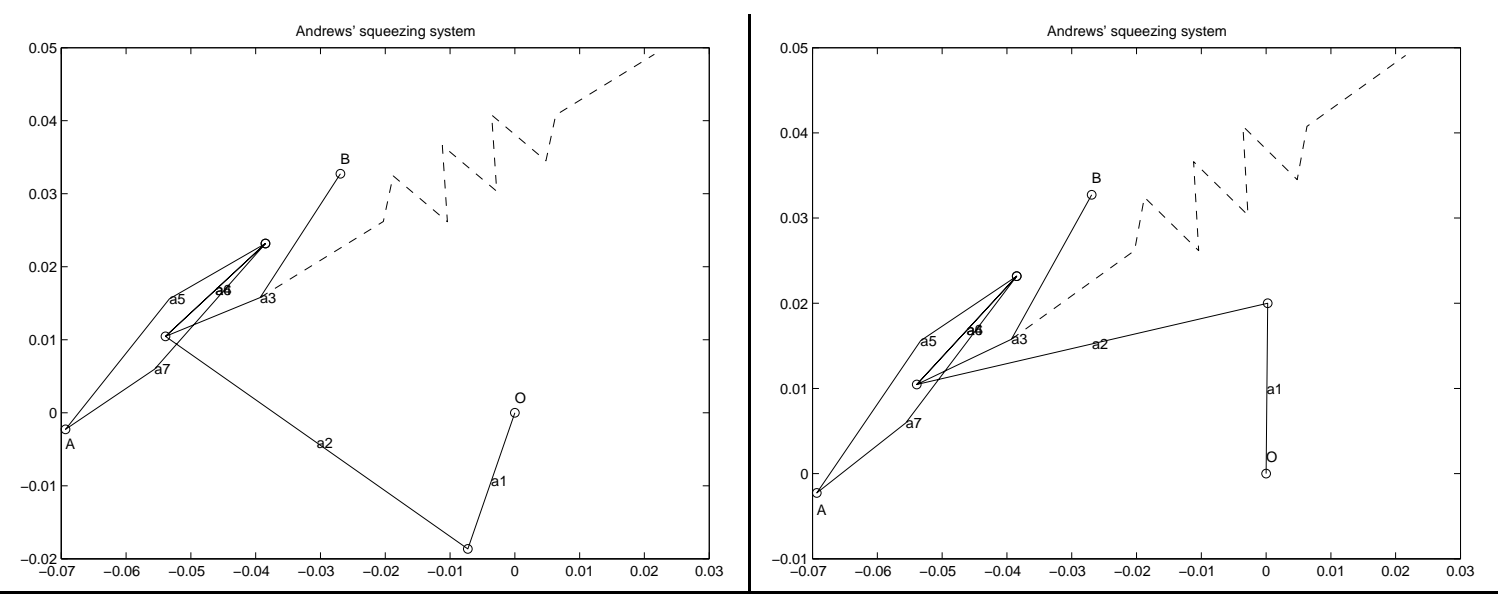

Figure 8: Singular positions (according to $J_{367}, U_{1}$ ) when $b_{1}=-0.02691, a_{1}=0.02, a_{2}=$ 0.055 and $a_{3}, \ldots, a_{7}$ have the original values. The physical interpretation is as in Figure 5.

\subsection{Appendix}

The coefficients $f_{i}$ : The coefficients $f_{1}, \ldots, f_{5}$ in the context of $T_{5}$ are

$$
\begin{aligned}
f_{1} & =4\left(a_{5}-a_{4}\right)^{2}\left(b_{1}^{2}-2 b_{1} w_{1}+b_{2}^{2}-2 b_{2} w_{2}+w_{1}^{2}+w_{2}^{2}\right) \\
& =4\left(a_{5}-a_{4}\right)^{2}|b-w|^{2}, \\
f_{2} & =4\left(w_{1}-b_{1}\right)\left(a_{4}-a_{5}\right)\left(-b_{1}^{2}+2 b_{1} w_{1}-b_{2}^{2}+2 b_{2} w_{2}-w_{1}^{2}-w_{2}^{2}+a_{3}^{2}-a_{4}^{2}+2 a_{4} a_{5}-a_{5}^{2}\right) \\
& =4\left(w_{1}-b_{1}\right)\left(a_{4}-a_{5}\right)\left(a_{3}^{2}-\left(a_{4}-a_{5}\right)^{2}-|b-w|^{2}\right), \\
f_{3} & =b_{1}^{2}-2 b_{1} w_{1}+b_{2}^{2}-2 b_{2} w_{2}+2 b_{2} a_{4}-2 b_{2} a_{5}+w_{1}^{2}+w_{2}^{2}-2 w_{2} a_{4}+2 w_{2} a_{5}-a_{3}^{2}+a_{4}^{2}-2 a_{4} a_{5}+a_{5}^{2} \\
& =|b-w|^{2}+2\left(b_{2}-w_{2}\right)\left(a_{4}-a_{5}\right)-a_{3}^{2}+\left(a_{4}-a_{5}\right)^{2}, \\
f_{4} & =b_{1}^{2}-2 b_{1} w_{1}+b_{2}^{2}-2 b_{2} w_{2}-2 b_{2} a_{4}+2 b_{2} a_{5}+w_{1}^{2}+w_{2}^{2}+2 w_{2} a_{4}-2 w_{2} a_{5}-a_{3}^{2}+a_{4}^{2}-2 a_{4} a_{5}+a_{5}^{2} \\
& =|b-w|^{2}-2\left(b_{2}-w_{2}\right)\left(a_{4}-a_{5}\right)-a_{3}^{2}+\left(a_{4}-a_{5}\right)^{2}, \\
f_{5} & =a_{3}^{2}-a_{4}^{2}+2 a_{4} a_{5}-a_{5}^{2}-b_{1}^{2}+2 b_{1} w_{1}-b_{2}^{2}+2 b_{2} w_{2}-w_{1}^{2}-w_{2}^{2} \\
& =a_{3}^{2}-\left(a_{4}-a_{5}\right)^{2}-|b-w|^{2} .
\end{aligned}
$$




\begin{tabular}{|c|c|c|}
\hline variables & singularity 1 & singularity 2 \\
\hline$c_{1}$ & -0.3621 & 0.0127 \\
\hline$s_{1}$ & -0.9322 & 0.9999 \\
\hline$c_{2}$ & 0.1860 & 0.1860 \\
\hline$s_{2}$ & 0.9862 & -0.9826 \\
\hline$c_{3}$ & 0.6364 & 0.6364 \\
\hline$s_{3}$ & -0.7714 & -0.7714 \\
\hline$c_{4}$ & 0 & 0 \\
\hline$s_{4}$ & -1 & -1 \\
\hline$c_{5}$ & 0.7714 & 0.7714 \\
\hline$s_{5}$ & 0.6364 & 0.6364 \\
\hline$c_{6}$ & 0 & 0 \\
\hline$s_{6}$ & 1 & 1 \\
\hline$c_{7}$ & -0.6364 & -0.6364 \\
\hline$s_{7}$ & 0.7714 & 0.7714 \\
\hline
\end{tabular}

Expressed in angles, these are

\begin{tabular}{|c|c|c|}
\hline Angles & singularity 1 & singularity 2 \\
\hline$y_{1}$ & -1.9413 & 1.5581 \\
\hline$y_{2}$ & 1.3837 & -1.3837 \\
\hline$y_{3}$ & -0.8810 & -0.8810 \\
\hline$y_{4}$ & 1.5708 & 1.5708 \\
\hline$y_{5}$ & 0.6898 & 0.6898 \\
\hline$y_{6}$ & 1.5708 & 1.5708 \\
\hline$y_{7}$ & 2.2606 & 2.2606 \\
\hline
\end{tabular}

Table 2: The singularities of $J_{367}$ type, original values apart from $b_{1}, a_{1}, a_{2}$. The values are presented only with 4 decimals but were computed with 16 decimals.

The coefficients $d_{i}, l_{i}$ : The coefficients $d_{i}, l_{i}$ in the context of $K_{2}$ are

$$
\begin{aligned}
d_{1} & =2 a_{1} a_{2}\left(a_{3}^{2}+2 a_{3} b_{1} s_{3}-2 a_{3} b_{2} c_{3}+b_{1}^{2}+b_{2}^{2}\right) \\
d_{2} & =-4 a_{1}^{2} a_{2}^{2} s_{2}^{2} \\
d_{3} & =-a_{1}^{4}+2 a_{1}^{2} a_{2}^{2}+a_{1}^{2} a_{3}^{2}+2 a_{1}^{2} a_{3} b_{1} s_{3}-2 a_{1}^{2} a_{3} b_{2} c_{3}+a_{1}^{2} b_{1}^{2} \\
& +a_{1}^{2} b_{2}^{2}-a_{2}^{4}+a_{2}^{2} a_{3}^{2}+2 a_{2}^{2} a_{3} b_{1} s_{3}-2 a_{2}^{2} a_{3} b_{2} c_{3}+a_{2}^{2} b_{1}^{2}+a_{2}^{2} b_{2}^{2} \\
& =-2 a_{1} a_{2}\left(a_{3}^{2}+2 a_{3} b_{1} s_{3}-2 a_{3} b_{2} c_{3}+b_{1}^{2}+b_{2}^{2}\right) \\
l_{1} & =2 a_{1} a_{2}\left(a_{3} s_{3}+b_{1}\right) \\
l_{2} & =-\left(a_{3} c_{3}-b_{2}\right)\left(a_{1}^{2}-a_{2}^{2}+a_{3}^{2}+2 a_{3} b_{1} s_{3}-2 a_{3} b_{2} c_{3}+b_{1}^{2}+b_{2}^{2}\right) \\
l_{3}=\left(a_{3} c_{3}-b_{2}\right) a_{2} s_{2}-\left(a_{3} s_{3}+b_{1}\right) a_{1} . &
\end{aligned}
$$




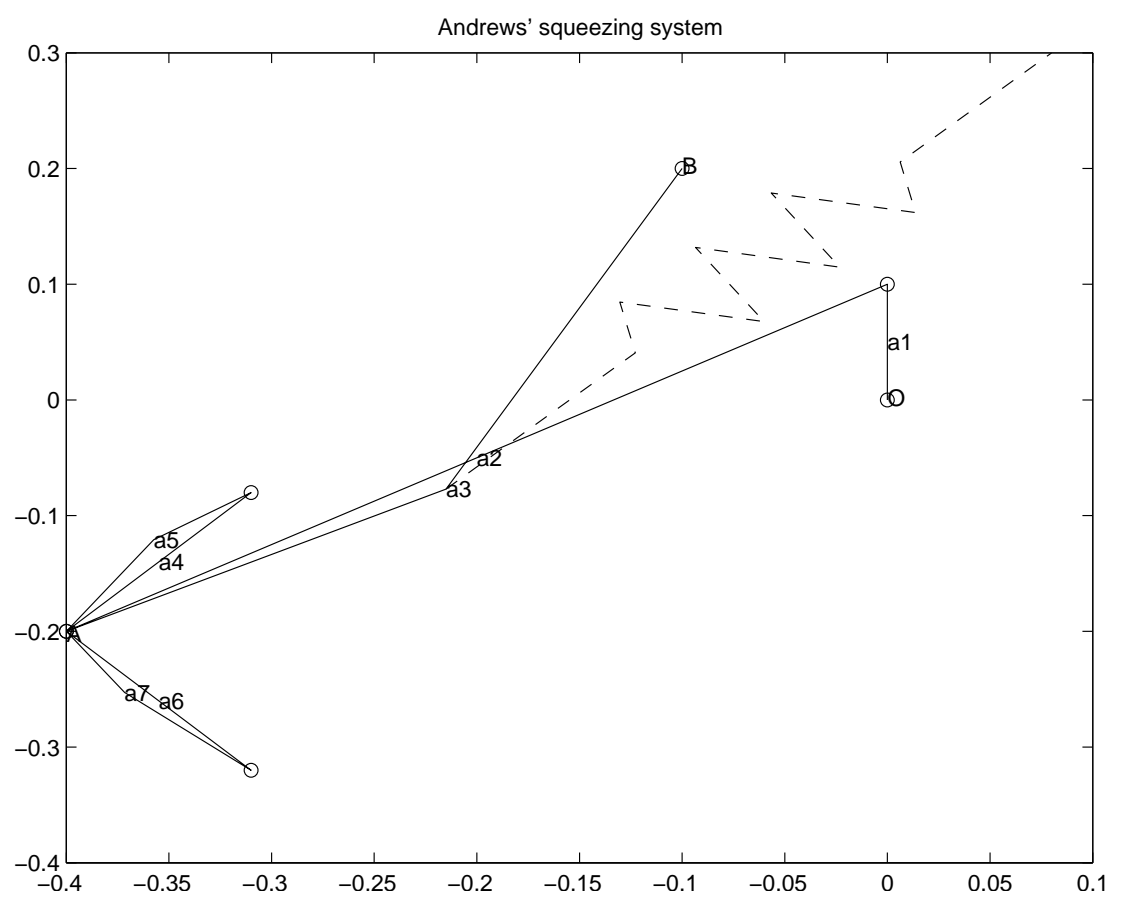

Figure 9: A singular configuration with rational $c_{i}, s_{i}, a_{i}, b_{i}$.

We can also simplify these expressions:

$$
\begin{aligned}
d_{0} & =a_{3}^{2}+|b|^{2}+2 a_{3}\left(b_{1} s_{3}-b_{2} c_{3}\right) \\
d_{1} & =2 a_{1} a_{2} d_{0} \\
d_{2} & =n_{1} n_{2} \\
d_{3} & =\left(a_{1}^{2}+a_{2}^{2}\right) d_{0}-\left(a_{1}^{2}-a_{2}^{2}\right)^{2} \\
n_{1} & =\left(a_{1}+a_{2}\right)^{2}-d_{0} \\
n_{2} & =\left(a_{1}-a_{2}\right)^{2}-d_{0}=4 a_{1} a_{2}-n_{1} \\
l_{1} & =-d_{1} \\
l_{3} & =-\left(a_{3} c_{3}-b_{2}\right)\left(a_{1}^{2}-a_{2}^{2}+d_{0}\right) \\
l_{4} & =-\left(a_{3} s_{3}+b_{1}\right)\left(a_{2} c_{2}+a_{1}\right)+a_{2} s_{2}\left(a_{3} c_{3}-b_{2}+2 a_{1} s_{1}\right) \\
\hat{g}_{1} & =-4 a_{1}^{2} a_{2}^{2} s_{2}^{2}+n_{1}\left(4 a_{1} a_{2}-n_{1}\right) \\
\hat{g}_{2} & =2 a_{1} a_{2} d_{0} c_{2}+\left(a_{1}^{2}+a_{2}^{2}\right) d_{0}-\left(a_{1}^{2}-a_{2}^{2}\right)^{2} \\
\hat{g}_{3} & =-2 a_{1} a_{2} d_{0} s_{1}+2 a_{1} a_{2}\left(a_{3} s_{3}+b_{1}\right)-\left(a_{3} c_{3}-b_{2}\right)\left(a_{1}^{2}-a_{2}^{2}+d_{0}\right) \\
\hat{g}_{4} & =\left(a_{1}^{2}-a_{2}^{2}\right) c_{1}+l_{4}
\end{aligned}
$$


The coefficients $r_{i}$ : The coefficients $r_{i}$ in the context of $L_{1}$ are

$$
\begin{aligned}
r_{1} & =\left(a_{1}^{2}+a_{2}^{2}\right)|b|^{2}-2 b_{1} a_{1}^{2} a_{3} s_{7}-2 b_{1} a_{2}^{2} a_{3} s_{7}+2 b_{2} a_{1}^{2} a_{3} c_{7}+2 b_{2} a_{2}^{2} a_{3} c_{7}-\left(a_{1}^{2}-a_{2}^{2}\right)^{2}+\left(a_{1}^{2}+a_{2}^{2}\right) a_{3}^{2} \\
r_{2} & =2 a_{1}\left(b_{1} a_{2}-a_{2} a_{3} s_{7}\right) s_{2} \\
r_{3} & =b_{1}^{2} b_{2}+b_{1}^{2} a_{3} c_{7}-2 b_{1} b_{2} a_{3} s_{7}-2 b_{1} a_{3}^{2} c_{7} s_{7}+b_{2}^{3}+3 b_{2}^{2} a_{3} c_{7}+b_{2} a_{1}^{2}-b_{2} a_{2}^{2}+3 b_{2} a_{3}^{2} c_{7}^{2} \\
& +b_{2} a_{3}^{2} s_{7}^{2}+a_{1}^{2} a_{3} c_{7}-a_{2}^{2} a_{3} c_{7}+a_{3}^{3} c_{7} \\
r_{4} & =\left(2 a_{1} a_{2}\right) s_{1} s_{2}+\left(-b_{1} a_{2}+a_{2} a_{3} s_{7}\right) c_{2}+\left(-b_{2} a_{2}-a_{2} a_{3} c_{7}\right) s_{2}+\left(-b_{1} a_{1}+a_{1} a_{3} s_{7}\right)
\end{aligned}
$$

\section{References}

[Arp01] T. Arponen. Regularization of constraint singularities in multibody systems. Multibody System Dynamics, 6(4):355-375, 2001.

[BA94] E. Bayo and A. Avello. Singularity-free augmented Lagrangian algorithms for constrained multibody dynamics. Nonl. Dyn., 5:209-231, 1994.

[CLO92] D. Cox, J. Little, and D. O'Shea. Ideals, Varieties and Algorithms. Springer, Berlin, 1992.

[EH95] E. Eich and M. Hanke. Regularization methods for constrained mechanical multibody systems. Z. Angew. Math. Mech., 10:761-773, 1995.

[Eis96] D. Eisenbud. Commutative Algebra, volume 150 of Graduate Texts in Mathematics. Springer, 1996. corr. 2nd printing.

[Gil78] D.R.A Giles. A comparison of three problem-oriented simulation programs for dynamic mechanical systems. PhD thesis, University of Waterloo, Waterloo, Ontario, 1978.

[GP02] G.-M. Greuel and G. Pfister. A Singular introduction to commutative algebra. Springer-Verlag, Berlin, 2002. With contributions by Olaf Bachmann, Christoph Lossen and Hans Schönemann, With 1 CD-ROM (Windows, Macintosh, and UNIX).

[GPS05] G.-M. Greuel, G. Pfister, and H. Schönemann. Singular 3.0. A Computer Algebra System for Polynomial Computations, Centre for Computer Algebra, University of Kaiserslautern, 2005. http://www.singular.uni-kl.de.

[HW91] E. Hairer and G. Wanner. Solving Ordinary Differential Equations II: stiff and differential-algebraic problems, volume 14 of Computational Mathematics. Springer, 1991.

[LR05] B. Leimkuhler and S. Reich. Simulating Hamiltonian Dynamics. Cambridge University Press, 2005.

[Man81] D.W. Manning. A computer technique for simulating dynamic multibody systems based on dynamic formalism. PhD thesis, University of Waterloo, Waterloo, Ontario, 1981. 
[McC00] J.M. McCarthy. Geometric Design of Linkages, volume 11 of Interdisciplinary Applied Mathematics. Springer, New York, 2000.

[MI03] F. Mazzia and F. Iavernaro. Test set for initial value problem solvers. Release 2.2, Department of Mathematics, University of Bari, August 2003. http://pitagora.dm.uniba.it/ testset/.

[Nor76] D. Northcott. Finite Free Resolutions, volume 71 of Cambridge Tracts in Mathematics. Cambridge Univ. Press, 1976.

[RS88] R. Roberson and R. Schwertassek. Dynamics of Multibody Systems. Springer, 1988.

[Sch90] W. Schiehlen, editor. Multibody Systems Handbook. Springer, Berlin, 1990. 